\title{
Size and roughness dependent temperature effects on surface charge of silica nanoparticles
}

\author{
B. Oyku Alan, Murat Barisik \\ Department of Mechanical Engineering, Izmir Institute of Technology, IZMIR 35430, Turkey
}

\section{H I G H L I G H T S}

- Nanoparticle surface charge increases with increasing temperature.

- Temperature effects on surface charge change with particle size and surface condition.

- Temperature dependent ionic diffusion and surface charge regulation were considered.

- Temperature dependent solution of PNP with charge regulation is presented.

- A model for nanoparticle charging is extended from analytical solution.

\section{A R T I C L E I N F O}

\section{Keywords:}

Surface charge density

Silica nanoparticles

Charge regulated surface

Nanoscale surface patterns/roughness

Temperature dependence

\section{G R A P H I C A L A B S T R A C T}

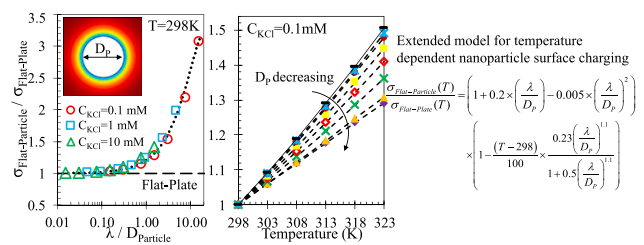

\begin{abstract}
A B S T R A C T
Silica nanoparticles (SNP) with different sizes and surface areas are used in numerous micro/nanofluidic applications, while their surface charge properties play a major role in their function. In many of these applications, SNPs also undergo temperature variation. We present that an increase in temperature yields a substantial increase in SNP surface charge depending on nanoparticle size and surface roughness, which cannot be estimated by existing theory. As a continuation of our earlier work characterizing the deviation of SNP surface charging from theoretical predictions due to curvature and EDL overlap effects, this study presents the differentiation from the theory in temperature dependence under various conditions. As we calculate surface chemistry as a function of local ionic conditions (Charge Regulation), temperature variation changed the equilibrium constants of protonation/deprotonation reactions of the SNP surface, in addition to changes occurring in relative permittivity and ionic mobilities. Results show that variation of SNP surface charge by temperature decreases by decreasing particle size and/or increasing roughness size, compare to theoretical flat plate calculations considering similar temperature-dependent properties and charge regulation on the surface. We characterized these deviations by obtaining an "electrokinetic similarity" between different systems of various size and roughness at various ionic conditions based on the non-dimensional groups of $\lambda / D_{P}$ and $\lambda / D_{R}$. Based on these, we devised a phenomenological model as an extension to the flat plate theory to successfully predict the surface charge of SNPs as a function of the particle size, roughness size, and temperature. The current findings are important for the characterization of SNPs through temperature variations and can also be used to adjust the surface charge of SNPs by tuning the temperature.
\end{abstract}

\footnotetext{
* Corresponding author.

E-mail address: muratbarisik@iyte.edu.tr (M. Barisik).
} 
Surface electric property of silica nanoparticles (SNP) plays a key role in numerous micro/nanofluidic applications including bio-imaging $[1,2]$, bio-sensing [3], bio-catalysis [4,5], drug delivery [6-8], and gene delivery [9-11]. During these processes, variation in temperature develops due to various forms of heat dissipation, e.g. Joule heating. By changing temperature, physical properties of the surrounding electrolyte as well as the surface chemistry of the particle undergo a temperature-dependent behavior which creates significant variation in the surface charging of SNPs.

Strong temperature variation develops, for example, during capillary electrophoresis due to Joule heating when an electric field is applied through an electrolyte solution. During electrophoresis, the viscosity and permittivity of the liquid medium, the diffusivity of ionic species, and physicochemical reactions on the particle surface vary by temperature. Multiple studies focused on the temperature dependent electrophoretic behavior of SNPs. For example, temperature effects on capillary electrophoresis as an increasing sample velocity is described theoretically [12], numerically [13], and experimentally [14]. While the variation of surface electric properties appears a complication to be considered for a successful electrophoresis-based application, it also emerges as a mechanism to control/manipulate the associated physics. This idea was implemented in a nanofluidic gating mechanism known as "Thermal Gate" where heating up and cooling down channel surface controls the ionic transport [15]. Similarly, temperature has been found to be a key determinant in salinity-gradient-driven energy conversion processes [16]. Simply, temperature increases the ionic transport by increasing the ionic mobility and silica surface charge [15,17-19]. Hence, multiple studies have been dedicated to investigate temperature dependent variation of surface charge of silica. Overall, silica surface charge is found to increase by increasing temperature as a result of the change in the association/disassociation reactions and ion adsorption on the surface [17]. Such response of surface physicochemistry to temperature change can be captured only by considering so-called "Charge Regulation (CR)" nature of the surfaces [20]. CR has been observed by multiple experiments in surface force measurements by colloids [21,22] and AFM $[23,24]$. Since then, researchers have attempted to apply CR into calculations using active charge models as boundary conditions to resolve surface charging of silica nano-channels [25-29], nano-pores [30-33], mesoporous systems [34,35], and nanoparticles [36-38]. Specific for temperature dependence, Hsu et al. focused on a $20 \mathrm{~nm} \mathrm{SNP}$ and showed that its electrophoretic mobility increases up to $40 \%$ with a $5 \mathrm{~K}$ increase in temperature. However, temperature dependent surface charging of SNPs at different particle sizes and surface conditions has not yet been described.

With the help of recent techniques, various forms of SNPs with different particle sizes and surface conditions have been synthesized. For such nano-systems, particle size and surface condition determine the local variation of the ionic concentration as well as the resulting electric potential at the surface. First, a decrease in overall particle size develops curvature effects so that the ionic condition around the particle diverges from a flat surface. Curvature effects are due to the decreased surface to volume ratio from the decrease in particle diameter as observed through several studies $[36,39,40]$. Second, the surface structures of either naturally developed or engineered surface patterns/roughness create local variation of ionic distribution which differs from existing flat surface theory. In the case of a nanoparticle roughness composed of

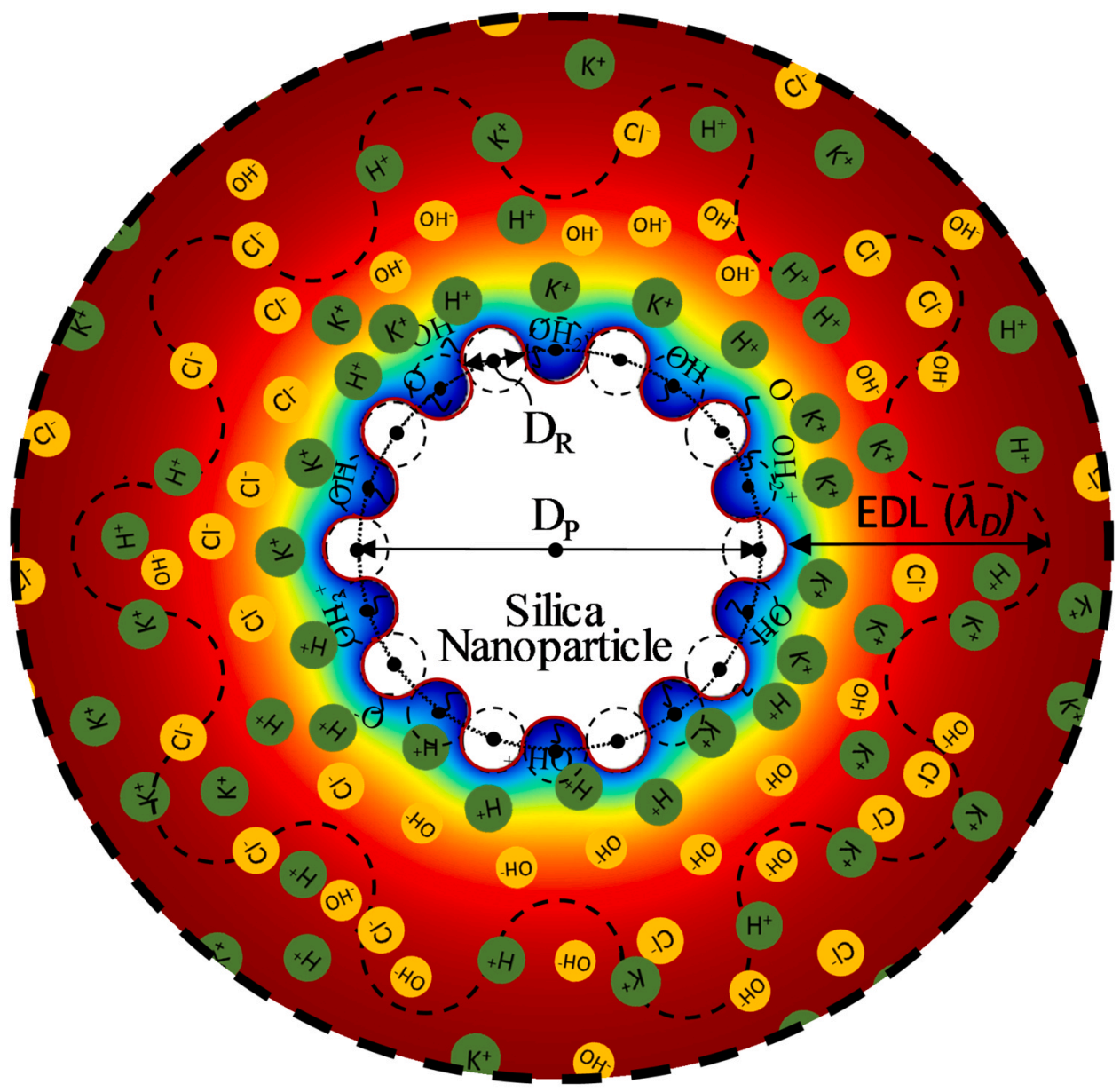

Fig. 1. Schematic illustration of the simulation domain consisting of patterned silica surface and four different ionic species with natural silanol groups of silica surface. 
valleys and hills, ionic layers in valleys extending from opposing convex surfaces overlap while curvature effects develop over the concave surfaces at the hills of the surface roughness. Such local variation of electric charge as a function of the surface nano topology was observed by a few studies through AFM measurements [41] and numerical simulations $[42,43]$ over planar surfaces. Recently, we studied the charge of SNPs and planar surfaces with varying size of surface nanopatterns/roughness $[44,45]$. While lower and higher charges were observed at the pits and the tips of surface pattern, the average of local surface charges became lower than the theoretical predictions. Based on the numerical calculations, phenomenological models were developed as an extension to the existing flat surface theory to predict the average surface charge on a nano patterned flat surface and SNP as a function of the surface pattern size, ionic concentration and $\mathrm{pH}$, and particle diameter $[44,45]$.

Our objective is to resolve temperature effects on SNP surface charge at different particle diameters and surface conditions. Poisson-NernstPlanck equations will be solved using a multi-ion charge regulation model $[36,37]$. Surface protonation and deprotonation reactions at the solid/liquid interface will be considered using the active charge model defined on surface boundaries. Temperature dependent variation in permittivity, ionic mobility, and equilibrium constants of the surface reactions are assessed coupled with CR calculations. We will provide general characterization for temperature dependent surface charge behavior of SNPs. We will test the solution of current theoretical calculations and develop extended phenomenological models to correct them.

\section{Theoretical background and mathematical model}

We considered a rough silica nanoparticle with diameter $\mathrm{D}_{\mathrm{P}}$ while a representative nano-roughness/pattern in the form of repeating concave and convex circles were formed as a function of roughness diameter $D_{R}$, as illustrated in Fig. 1. This surface pattern allows surface structures to be controlled by simply varying the single quantity $D_{R}$ and perform a parametric study by systematically varying it. Furthermore, the charging mechanisms developing on the circular pattern are similar to the flat surface nanoparticles previously studied [44] and validated with our earlier work [45]. The liquid phase is considered as $\mathrm{KCl}$ (i.e. symmetric 1:1) aqueous electrolyte solution consists of 4 types of ionic species namely; $\mathrm{H}^{+}, \mathrm{K}^{+}, \mathrm{Cl}^{-}$and $\mathrm{OH}^{-}$ions with their bulk values being $c_{10}, c_{20}, c_{30}$, and $c_{40}$, respectively. These bulk concentrations are taken as: $c_{10}=10^{-p H+3}$, and $c_{40}=10^{-(14-p H)+3}$ while $c_{20}=c_{K C l}$, and $c_{30}$ $=c_{K C l}+c_{10}-c_{40}$ for $\mathrm{pH}<7$, or $c_{20}=c_{K C l}+c_{10}-c_{40}$, and $c_{30}=c_{K C l}$ for $\mathrm{pH}>7[30,46-48]$.

When a solid surface meets with an electrolyte, protonation/deprotonation reactions develops on the solid/liquid interface such that the surface will be charged due to surface chemical reactions and adsorption of ions. Surface charge creates an ionic accumulation on the surface. Generally, ionic distribution in surface normal direction can be defined with the Boltzmann distribution (BD) [49-51]. Combining with the Poisson equation, Poisson-Boltzmann (PB) equation describes the electric potential distribution through the ionic layering also called electric double layer (EDL). PB can be solved analytically under certain assumptions. First, the Debye-Hückel simplification [52] is required which is valid for surface electric potentials lower than $25 \mathrm{mV}$. Second, surfaces should be flat without any curvature effects and overlap of EDLs of opposing surfaces. However, the electric potential of SNPs can get values higher than $25 \mathrm{mV}$ while particle diameters smaller than $50 \mathrm{~nm}$ develop curvature effects and surface structures develop EDL overlaps. For this case, instead of Boltzmann Distribution, the Nernst-Planck equation should be used to calculate ionic mass transport that we will employ Poisson-Nernst-Planck (PNP) equations in our calculations. First, electric potential distribution within the EDL obeys the Poisson equation that is presented in Eq. (1). In this equation, $\psi$ is the electric potential, $\varepsilon_{0}$ is the permittivity of vacuum, $\varepsilon_{\mathrm{r}}$ is the relative permittivity of the electrolyte solution, $F$ is the Faraday constant, and $z_{i}$, is the valence of the $i$ th ionic species $\left(\mathrm{i}=1\right.$ for $\mathrm{H}^{+} ; \mathrm{i}=2$ for $\mathrm{K}^{+} ; \mathrm{i}=3$ for $\mathrm{Cl}^{-} ; \mathrm{i}=4$ for $\left.\mathrm{OH}^{-}\right)$respectively.

$-\varepsilon_{0} \varepsilon_{r} \nabla^{2} \psi=F\left(z_{i} c_{i}\right)$

The extension of EDL from surface is characterized by the debye length $(\lambda)$. Debye length can be evaluated using Eq. (2). Boltzmann and Avogadro constants are denoted by $k_{B}$ and $N_{A}$, respectively while $T$ is the temperature and $e$ is the elementary charge.

$\lambda=\frac{1}{\kappa}=\sqrt{\frac{\varepsilon_{0} \varepsilon_{r} k_{B} T}{N_{A} e^{2} \sum c_{i} z_{i}{ }^{2}}}$

Next, ionic mass transport is governed by Nernst-Planck equation as shown in Eq. (3). Here, $\vec{N}_{i}$ is the flux density, $R$ is the universal gas constant, $D_{i}$ is the diffusivity, and $T$ is the temperature. Concentrations of ionic species are maintained at their bulk values at far ends of the reservoir domain and no flux of ionic species is allowed through the SNP surface.

$\nabla \cdot \vec{N}_{i}=\nabla \cdot\left(-D_{i} \nabla c_{i}-z_{i} \frac{D_{i}}{R T} F c_{i} \nabla \psi\right)=0$

Combination of Eqs. (1) and (3) forms the steady-state PNP equations. As a surface boundary condition, constant surface charge $[9,53]$, or constant potential [54] are frequently employed in the solution of PNP equations in the literature. However, enforcing constant surface charge or potential at the interface does not represent physical response of the surface chemistry. The surface reactions develop based on the local ionic environment at the interface that neither surface charge nor potential remain constant. Instead, both of them undergo variation in response to the variation in ionic distribution and create a new equilibrium accordingly. In this case, the nature of surface psychochemistry should be calculated using a proper CR model. We employed the previously developed multi-ion charge-regulation model $[36,37]$. Hence, electric potential and surface charge density on the surface are coupled as,

$-\varepsilon_{0} \varepsilon_{r} \vec{n} \cdot \nabla \psi=\sigma_{w}$

where $\sigma_{\mathrm{w}}$ is the surface charge density. Surface charge density is defined by an active surface charge model to calculate CR nature of the silica as a function of surface psychochemistry. Silica nanoparticle's surface in contact with an electrolyte solution is charged due to protonation/ deprotonation of ions. We considered the following two fundamental dissociation/association reactions at the solid/liquid interface as:

$\mathrm{SiOH} \leftrightarrow \mathrm{SiO}^{-}+\mathrm{H}^{+}$

$\mathrm{SiOH}+\mathrm{H}^{+} \leftrightarrow \mathrm{SiOH}_{2}^{+}$

The corresponding equilibrium constants can be calculated as:

$K_{A}=\frac{\Gamma_{S i O^{-}}\left[H^{+}\right]_{w}}{\Gamma_{S i O H}}, K_{B}=\frac{\Gamma_{S_{i O H}^{+}}}{\Gamma_{S i O H}\left[H^{+}\right]_{w}}$

where $\Gamma_{\mathrm{SiO}^{-}}, \Gamma_{\mathrm{SiOH}}$ and $\Gamma_{\mathrm{SiOH}_{2}^{+}}$are the surface site densities of $\mathrm{SiO}-\mathrm{SiOH}$ and $\mathrm{SiOH}_{2}^{+}$, respectively and $\left[\mathrm{H}^{+}\right]_{w}$ is the hydrogen concentration at the solid/liquid interface. Hence, the surface charge density of the silica surface can be defined as:

$\sigma_{w}=-\frac{F \Gamma_{\text {total }}}{N_{A}} \frac{K_{A}-K_{B}\left[H^{+}\right]^{2}{ }_{w}}{K_{A}+\left[H^{+}\right]_{w}+K_{B}\left[H^{+}\right]^{2}{ }_{w}}$

Some of the parameters of the above listed equations develop substantial variation by temperature. First, in the description of the motion of a charged chemical species in a fluid medium, relative permittivity and ionic diffusivity of the $\mathrm{KCl}$ aqueous solution show temperature dependence. As we aim to study a relatively dilute aqueous $\mathrm{KCl}$ solution, corresponding properties are assumed to be same as those of the pure 
Table 1

Limiting ionic conductance values at $298.15 \mathrm{~K}$ and model parameters of Eq. (11) to describe temperature dependence.

\begin{tabular}{|c|c|c|c|c|}
\hline $\begin{array}{l}\text { Ionic species } \\
\text { (i) }\end{array}$ & $\begin{array}{l}\lambda_{i}^{0} @ 298.15\left(\mathrm{~S} \times \mathrm{cm}^{2} \times \mathrm{mol}^{-}\right. \\
1)\end{array}$ & $\alpha_{i 1}$ & $\alpha_{i 2} \times 10^{2}$ & $\alpha_{i 3} \times 10^{4}$ \\
\hline$H^{+}$ & 349.85 & 4.81595 & -1.03125 & -0.7670 \\
\hline $\mathrm{OH}^{-}$ & 199.20 & 3.52031 & 0.82700 & 0.7120 \\
\hline$K^{+}$ & 73.50 & - & - & - \\
\hline $\mathrm{Cl}^{-}$ & 76.35 & 1.54037 & 0.46500 & -0.1285 \\
\hline
\end{tabular}

water. The temperature dependence of relative permittivity can be expressed as [55-57]:

$\varepsilon_{r}(T)=\exp \left[4.47615-4.60128 \times 10^{-3} \times \Delta T+2.6952 \times 10^{-7} \times \Delta T^{2}\right]$

where $\Delta T=T-273.15$. Next, the temperature dependence of diffusivity of each ionic species can be calculated by Nernst-Haskell equation $[18,58]$ :

$D_{i}=\frac{R T}{F^{2}}\left(\frac{\lambda_{i}^{0}}{\left|z_{i}\right|}\right)$

where $F$ is the Faraday constant and, $\lambda_{i}^{0}$ and $z_{i}$ are the limiting ionic conductance and valence of ionic species $i$. The $\lambda_{i}^{0}$ ions in aqueous solution show a strong temperature dependence [59], which can be calculated as:

$\lambda_{i}^{0}=\lambda_{i @ 298.15}^{0}+\alpha_{i 1} \times \Delta T+\alpha_{i 2} \times \Delta T^{2}+\alpha_{i 3} \times \Delta T^{3}$

where $\lambda_{i}^{0} @ 298.15$ is the limiting conductance at $T=298.15 \mathrm{~K}$ and $\Delta T=$ $T-298.15$. The corresponding values of the parameters $\lambda_{i}^{0} @ 298.15, a_{i 1}$, $a_{i 2}$ and $a_{i 3}$ for $\mathrm{H}^{+}, \mathrm{OH}^{-}$and $\mathrm{Cl}^{-}$ions can be found from the literature as tabulated in Table $1[10,60,61]$.

However, the model parameters for $\mathrm{K}^{+}$do not exist in literature. For such a case, we turned to available experimental measurements. Table 2

Table 2

Experimental measurements for the limiting equivalent conductance of $\mathrm{K}^{+}$and $\mathrm{Cl}^{-}$in water at different temperatures from 0 to $100{ }^{\circ} \mathrm{C}$ from Robinson and Stokes [62].

\begin{tabular}{lllllllll}
\hline & $20{ }^{\circ} \mathrm{C}$ & $25{ }^{\circ} \mathrm{C}$ & $30{ }^{\circ} \mathrm{C}$ & $35{ }^{\circ} \mathrm{C}$ & $40{ }^{\circ} \mathrm{C}$ & $45^{\circ} \mathrm{C}$ & $50^{\circ} \mathrm{C}$ & $55^{\circ} \mathrm{C}$ \\
\hline$\lambda_{K}^{0}$ & 66.8 & 73.5 & 82.5 & 89.5 & 97.6 & 107.3 & 115.37 & 123.5 \\
$\lambda_{C l}^{0}$ & 68 & 76.35 & 84.6 & 91 & 99.2 & 108.5 & 117.1 & 125 \\
\hline
\end{tabular}

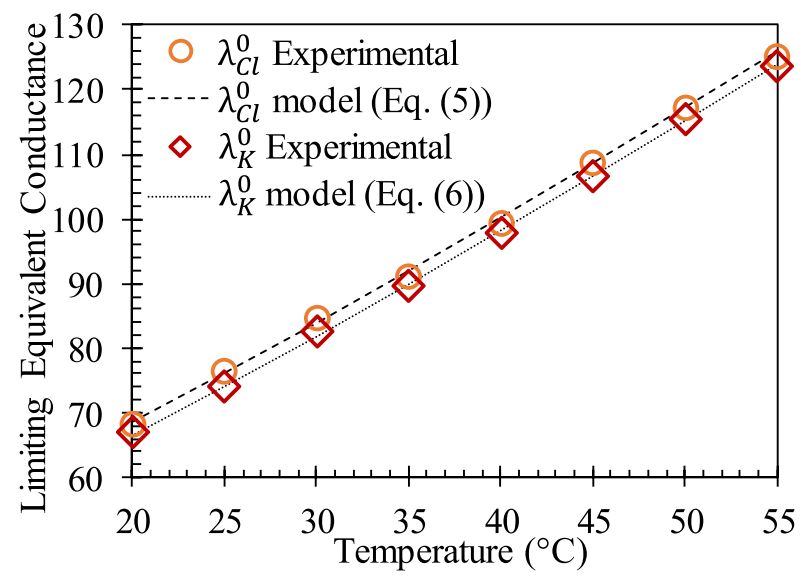

Fig. 2. Variation of limiting equivalent conductance values of $\mathrm{K}^{+}$and $\mathrm{Cl}^{-}$by temperature. Experimental data from Robinson and Stokes [62]. presents the experimental data [62]. With a slight difference, the limiting equivalent conductance of $\mathrm{K}^{+}$and $\mathrm{Cl}^{-}$are very similar to each other. We presented the data in Fig. 2.

Third degree polynomial models described as in Eq. (11) were applied to experimental data in Fig. 2. Since the variation of limiting equivalent conductance of $\mathrm{K}^{+}$and $\mathrm{Cl}^{-}$by temperature are very similar, we employed the available model parameters for $\mathrm{Cl}^{-}$by simply updating the corresponding limiting conductance at temperature $T=298.15 \mathrm{~K}$ $\left(\lambda_{i}^{0} @ 298.15\right)$. Eq. (12) provides successful descriptions of experimental measurements where $\Delta T=T-298.15$.

$\lambda_{C l}^{0}=76.35+1.54037 \times \Delta T+0.00465 \times \Delta T^{2}-0.1285 \times 10^{-4} \times \Delta T^{3}$

$\lambda_{K}^{0}=73.5+1.54037 \times \Delta T+0.00465 \times \Delta T^{2}-0.1285 \times 10^{-4} \times \Delta T^{3}$

Secondly, the equilibrium constants given in Eq. (7) show variation by temperature. Since the current study mostly focused on the behavior through the $\mathrm{pH}$ range of 5-9, where charge nature of the particle is dominated by $K_{A}$. This was also validated by titration experiments that proton adsorption on the surface is unlikely to occur in the normal $\mathrm{pH}$ range due to the low $\log K_{B}$ values [63]. So, we will neglect the temperature dependence of $K_{B}$ and use a constant value $\left(\mathrm{p} K_{B}=-\log K_{B}\right.$ $=1.9$ ) The temperature dependence of an equilibrium constant is defined by the Van't Hoff equation as a function of temperature and standard enthalpy change $\left(\Delta_{r} H\right)$ of the reaction as [64]:.

$\ln \frac{K\left(T_{2}\right)}{K\left(T_{1}\right)}=\frac{-\Delta_{r} H}{R}\left(\frac{1}{T_{2}}-\frac{1}{T_{1}}\right)$

Based on the Van't Hoff isotherm, temperature dependence of $K_{A}$ can be described as:

$\ln K_{A}=\frac{-\Delta_{r} G_{A}}{k_{B} T}$

where $\Delta_{r} G_{A}$ is the Gibbs energy change of the reaction which can be estimated as $9.60257 \mathrm{kcal} / \mathrm{mol}$ based on the reactions parameter for $T=300 \mathrm{~K}\left(p K_{A}=7, K_{A}=10^{-7}\right.$, and $K_{B}=0.0019872041 \mathrm{kcal} / \mathrm{mol} \mathrm{K}$ at $300 \mathrm{~K})$. For the current temperature range $(298-348 \mathrm{~K}) \Delta_{r} G_{A}$ is considered as a constant in Eq. (14) to describe temperature dependence of $K_{A}$.

Finally, Eqs. (1), (3) and (8) are coupled with the Eqs. (9), (10), (12), and (14) as,

$$
\begin{aligned}
& -\varepsilon_{0} \exp \left[4.47615-4.60128 \times 10^{-3} \times \Delta T+2.6952 \times 10^{-7} \times \Delta T^{2}\right] \nabla^{2} \psi \\
& \quad=F\left(z_{i} c_{i}\right)
\end{aligned}
$$

$$
\begin{aligned}
\nabla \cdot \vec{N}_{i}= & \nabla \cdot\left(-\frac{R T}{F^{2}}\left(\frac{\lambda_{i @ 298.15}^{0}+\alpha_{i 1} \times \Delta T+\alpha_{i 2} \times \Delta T^{2}+\alpha_{i 3} \times \Delta T^{3}}{\left|z_{i}\right|}\right) \nabla c_{i}\right. \\
& \left.-\frac{z_{i}}{F}\left(\frac{\lambda_{i @ 298.15}^{0}+\alpha_{i 1} \times \Delta T+\alpha_{i 2} \times \Delta T^{2}+\alpha_{i 3} \times \Delta T^{3}}{\left|z_{i}\right|}\right) c_{i} \nabla \psi\right) \\
= & 0
\end{aligned}
$$

$\sigma_{w}=-\frac{F \Gamma_{\text {total }}}{N_{A}} \frac{\exp \left[\frac{-\Delta_{r} G_{A}}{k_{B} T}\right]-K_{B}\left[H^{+}\right]^{2}{ }_{w}\left[\frac{-\Delta_{r} G_{A}}{k_{B} T}\right]+\left[H^{+}\right]_{w}+K_{B}\left[H^{+}\right]^{2}{ }_{w}}{\exp }$

Eqs. (15), (16) and (17) are solved using COMSOL Multiphysics software based on the finite element method. Fig. 3 explains steps of numerical calculations.

The solution procedure is validated by comparing its results with Hsu et al. [18], where surface electric potential of a $20 \mathrm{~nm}$ diameter flat (no roughness) silica particle immersed in $10^{-3} \mathrm{M} \mathrm{NaCl}$ solution is studied. We practiced our procedure using their parameters for $\mathrm{NaCl}$. In Fig. 4, surface electric potentials of a $20 \mathrm{~nm} \mathrm{SNP}$ at $\mathrm{pH}$ values of 7 and 9 


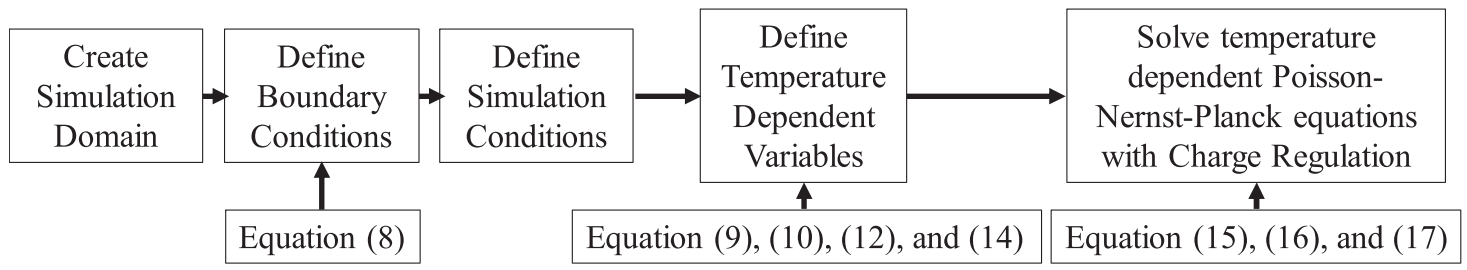

Fig. 3. Numerical flow chart of temperature dependent PNP with charge regulation calculations.
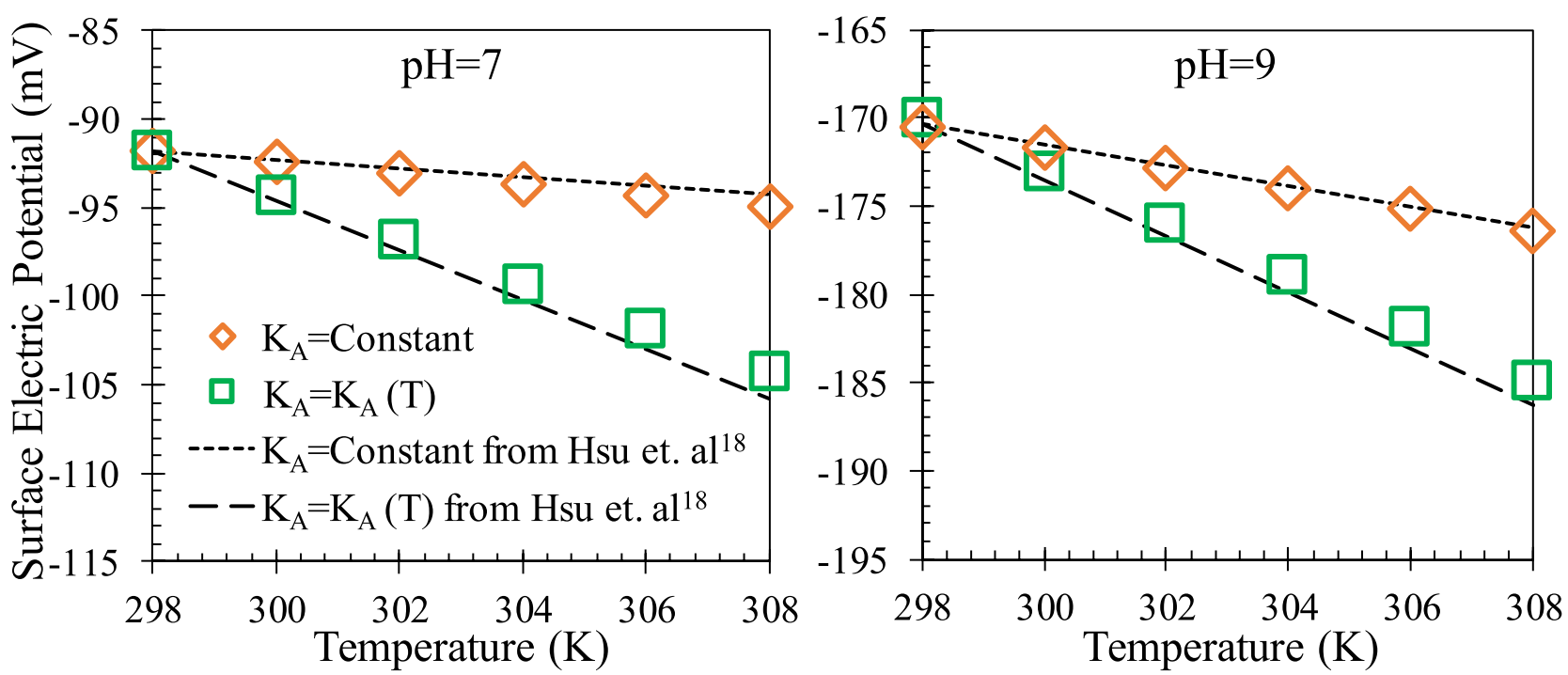

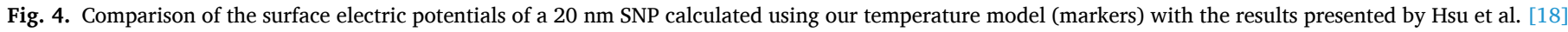

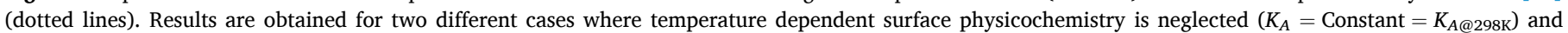
included $\left(K_{A}=K_{A}(T)\right)$.

calculated for two different cases. In the first case, temperature dependent surface physicochemistry is neglected so that $K_{A}$ is kept as a constant value in CR calculations. In the second case, we included variation of $K_{A}$ as a function of temperature using Eq. (14). Overall, the absolute values of surface electric potential of the SNP increases with an increase in temperature. While limited changes in surface potential are observed in the first case, a substantial increase develops when the temperature dependent $\mathrm{CR}$ is included. At $\mathrm{pH}=7$, surface electric potential increases more than $15 \%$ in the case of a $10 \mathrm{~K}$ increase of temperature when the temperature dependent $K_{A}$ is considered. A good agreement is observed between our calculations and the results of Hsu et al. [18]. This validation step also shows the importance of considering temperature dependent physicochemistry of CR in the characterization of SNP surface electric properties as a function of temperature.

As an addition, here, we also present the theoretical approximate solution for surface charging of a flat planar surface based on Poisson-Boltzmann solution derived by Yeh et al. [28]. Based on PB, Eq. (1) can be transformed into following form as,

$\frac{d^{2} \psi}{d x^{2}}=-\frac{1}{\varepsilon_{f} \varepsilon_{0}} \sum_{i=1}^{N} F z_{i} C_{i 0} \exp \left(-\frac{z_{i} F \psi}{R T}\right)$

An approximate solution for above given PB can be obtained by assuming the contributions of the ions $\mathrm{H}^{+}$and $\mathrm{OH}^{-}$to the net charge density is negligible. $\frac{d^{2}\left(\frac{z F \psi}{R T}\right)}{d x^{2}}=\kappa^{2} \sinh \left(\frac{z F \psi}{R T}\right)$

where $z=z_{2}=-z_{3}, C_{0}=C_{20}=C_{30}$ and $\lambda_{D}=\kappa^{-1}=\left(\varepsilon_{f} \varepsilon_{0} R T /\right.$ $\left.2 z^{2} F^{2} C_{0}\right)^{1 / 2}$ solution for a planar system yields the well-known Gouy-Chapman solution as,

$\psi=\frac{2 R T}{F z} \ln \left[\frac{1+\exp (-\kappa x) \tanh \left(\frac{F z \zeta}{4 R T}\right)}{1-\exp (-\kappa x) \tanh \left(\frac{F z \zeta}{4 R T}\right)}\right]$

For this solution, the concentration of $\mathrm{H}^{+}$ions at the solid/liquid interface is assumed to obey the Boltzmann distribution as,

$\left[H^{+}\right]_{s}=\left[H^{+}\right]_{0} \exp [(F \zeta) /(R T)]$

By substituting Eq. (21) into Eq. (8), we obtain,

$\sigma_{W}=-F N_{\text {total }}\left\{\frac{K_{A}-K_{B}\left[\left[H^{+}\right]_{0} \exp \left(-\frac{F \zeta}{R T}\right)\right]^{2}}{K_{A}+\left[H^{+}\right]_{0} \exp \left(-\frac{F \zeta}{R T}\right)+K_{B}\left[\left[H^{+}\right]_{0} \exp \left(-\frac{F \zeta}{R T}\right)\right]^{2}}\right\}$

Combining Eqs. (20) and (22) in Eq. (1) provides an analytical solution with charge regulation for silica surface charge as, 


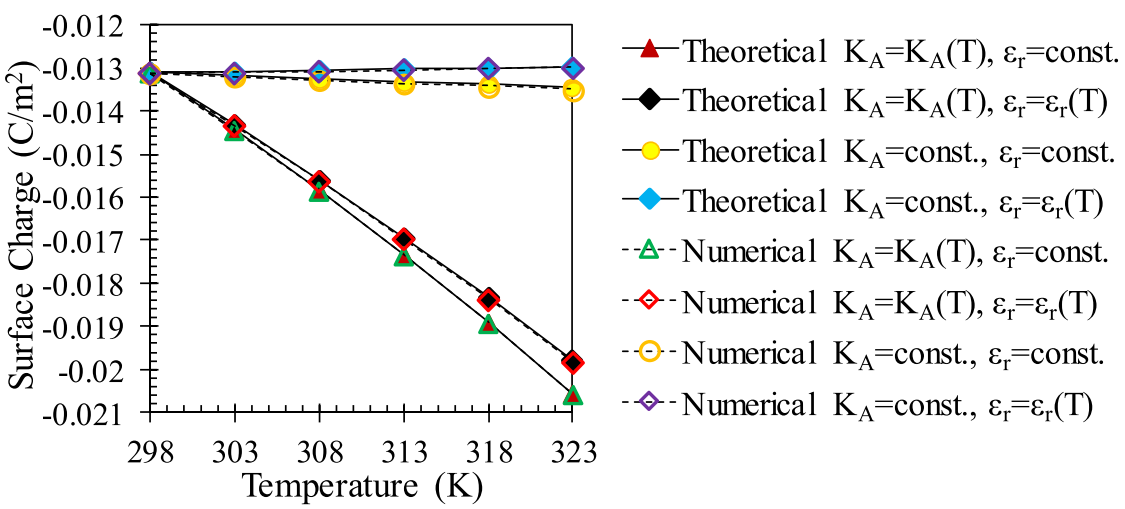

Fig. 5. The surface charge of a flat silica plate at different temperatures calculated theoretically using PB with CR from Yeh et al. (Eqs. (22) and (23) coupled with the Eq. (9), and (14)), and numerically using PNP with CR (Eqs. (1) and (3) coupled with the Eqs. (8), (9), (10), (12), and (14)). Constant values are at 298 K.
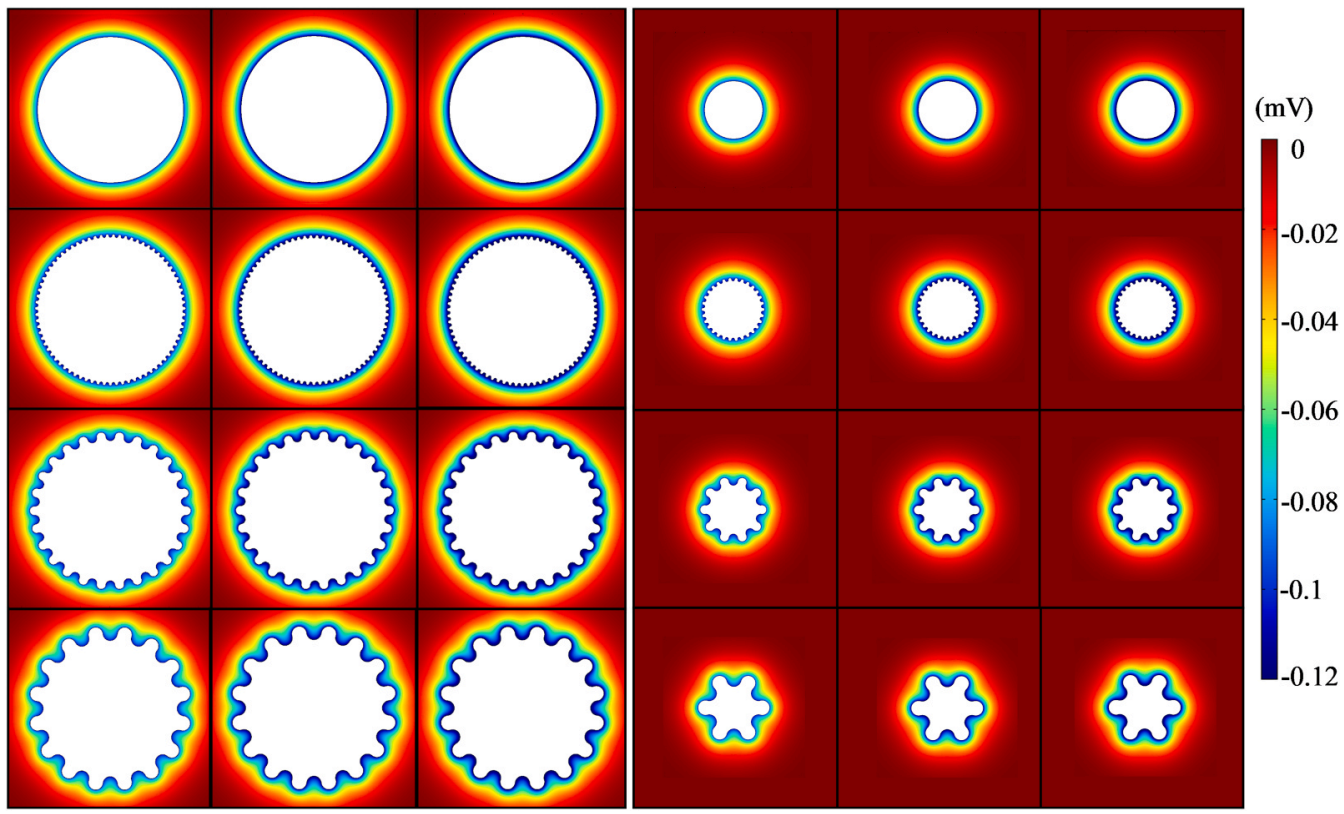

Fig. 6. Electric potential contours of different size nanoparticles at different roughness and temperatures. $\left(D_{P}=40,100 \mathrm{~nm}, D_{R}=0,2,6,10 \mathrm{~nm}, T=298\right.$, $313,323 \mathrm{~K})$.

$\frac{2 \varepsilon_{0} \varepsilon_{r} \kappa R T}{z F} \sinh \left(\frac{F z \zeta}{2 R T}\right)=-F N_{\text {total }}\left\{\frac{K_{A}-K_{B}\left[\left[H^{+}\right]_{0} \exp \left(-\frac{F \zeta}{R T}\right)^{2}\right]}{K_{A}+\left[H^{+}\right]_{0} \exp \left(-\frac{F \zeta}{R T}\right)+K_{B}\left[\left[H^{+}\right]_{0} \exp \left(-\frac{F \zeta}{R T}\right)\right]^{2}}\right\}$

This implicit approximation can be easily solved using a mathematical solver from MATLAB or Excel toolset. Once the zeta potential of surface, $\zeta$, is calculated, it can be substituted into Eq. (22) to calculate surface charge. Yeh et al. validated Eq. (23) by comparing its results with the results of numerical calculations of a full multi-ion model for all four ions and they obtained great agreement. Furthermore, Yeh et al. compared calculated surface charges for an isolated flat silica surface with the experimental data of Gaudin and Fuerstenau [65] which also showed a good agreement. We should underline here that the current model neglects the Stern layer. Existing literature mentioned that the Stern layer effect is significant for the $\mathrm{pH}$ range of 8-10, but for lower $\mathrm{pH}$ values, it seems it has nearly no effect on the surface charge [66].

We further implement temperature dependent parameters for both ionic transport and surface psychochemistry into calculations of Eq. (23). We systematically studied the contribution of each temperature dependent term in Fig. 5. We should underline here that Eq. (23) is only valid for a flat surface with an undisturbed EDL (no curvature and EDL overlap effects). The surface charge variation by temperature calculated theoretically for a flat planar surface provide further validation. But, more importantly, prediction of Eq. (23) provides a base to develop extended theories to predict SNP surface charging, which shows deviation from flat plate theory [28].

Temperature dependent variation of a planar silica surface is calculated both theoretically and numerically in Fig. 5. First, Eq. (23) has no consideration of diffusion as the PB equations use Boltzmann distribution to determine electric potential on the surface, instead of Nernst- 


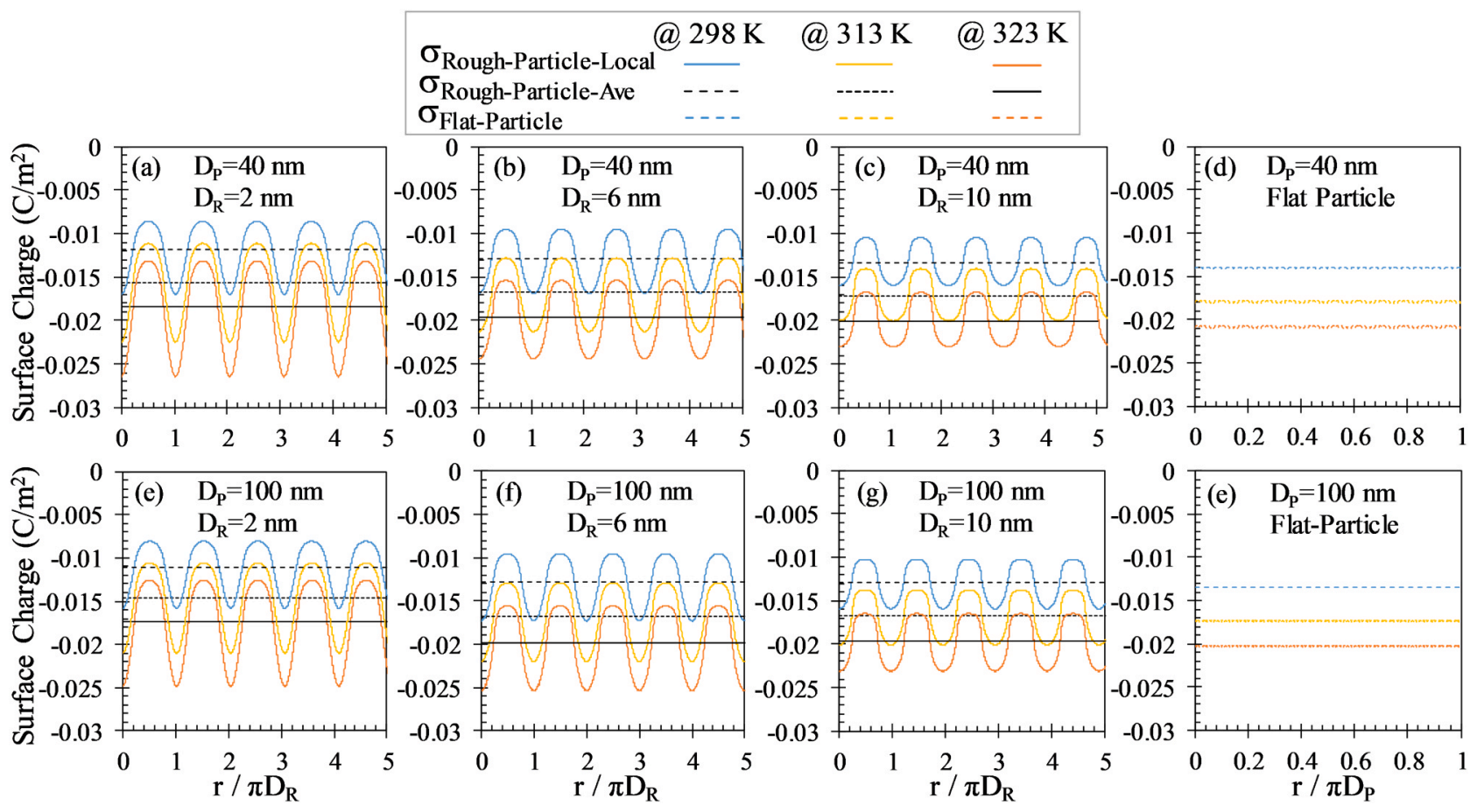

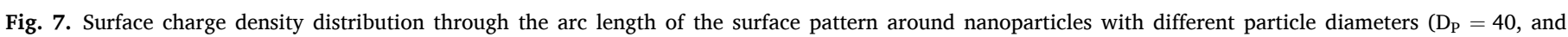
$100 \mathrm{~nm})$ and roughness diameters $\left(\mathrm{D}_{\mathrm{R}}=2,6\right.$, and $\left.10 \mathrm{~nm}\right)$ at different temperatures $(\mathrm{T}=298,313$, and $323 \mathrm{~K})$.

Planck employed by PNP. On the other hand, such an assumption seems appropriate for a flat planar surface case as observed from the comparison. Simply, theoretical calculations considering temperature dependent surface chemistry and relative permittivity yield identical results with the numerical solution of PNP with temperature dependent surface chemistry, relative permittivity, and ionic diffusivities. We also focused on the contribution of each temperature dependent parameter. Results show that relative permittivity has a negative effect on surface charge as the permittivity decreases with an increase in temperature. In the rest of the manuscript, we will present theory with $K_{A}=K_{A}(T), \varepsilon_{r}$ $=\varepsilon_{r}(T)$, which will be denoted as $\sigma_{\text {Flat-Plate }}$.

\section{Results and discussion}

We studied the electric potential distribution around the $40 \mathrm{~nm}$ and $100 \mathrm{~nm}$ diameter $\left(D_{\mathrm{P}}\right)$ nanoparticles at different temperatures of $298 \mathrm{~K}$, $313 \mathrm{~K}$ and $323 \mathrm{~K}$. Starting from a flat particle with no roughness, we added surface structures with roughness diameters $\left(D_{R}\right)$ of $2 \mathrm{~nm}, 4 \mathrm{~nm}$ and $10 \mathrm{~nm}$. Ionic conditions were at $\mathrm{C}_{\mathrm{KCl}}=1 \mathrm{mM}$ and $\mathrm{pH}=7$. In Fig. 6 , each column is at a constant temperature and each row has the same roughness diameter. As the temperature increase from left to right, the absolute value of the electric potential over the surfaces significantly increases as a result of both increased ionic activities and surface chemistry. As we discussed in Figs. 4 and 5, considering temperature dependence of ionic activities without including temperature dependence of charge regulation cannot predict such an increase. Here, temperature affects affected the relative permittivity, ionic mobilities, and equilibrium constant of surface reactions of the nanoparticle. From bottom to top, when the roughness diameter decreases, the gap between the roughness structures becomes smaller than the EDL thickness. As a result, electric double layers extending from opposite surfaces overlaps and ionic distribution shows local variation along the pattern surface. Interestingly, potential contours around the particle are almost perfectly circular, despite the patterned surface. In contrast, when the roughness diameter is bigger than the EDL thickness, overlap is diminished and the EDL grows as a projectile of the circular path of surface roughness so that potential contours around the particle shows the shape of the surface pattern. Next, when the particle diameter decreases from $100 \mathrm{~nm}$ to $40 \mathrm{~nm}$, EDL electric potential around the particle increases due to curvature effects. As a result of local variation of ionic distribution, surface electric properties also develop local variation described by the charge regulation nature of surface physicochemistry calculated by the active electric boundary condition. We should also mention that the PNP equation is not applicable to study system sizes comparable to molecular diameter. Hence, current calculation systematic based on PNP model is not appropriate to estimate surface charging for roughness sizes smaller than approximately $2 \mathrm{~nm}$ [29,67-69].

The resulted local surface charge densities are given in Fig. 7. The surface charge distribution on the SNP surfaces are plotted along the normalized radial position $\left(r / \pi D_{R}\right)$. In addition, we calculated the average of these local charges along the roughness for each case given as straight lines in the figures. Surface charges of the flat particle case are given at each temperature values along its perimeter which are also straight lines. We observed that surface charge around the rough nanoparticle varies along the patterned surface, but the variation repeats itself over every surface structure. Even though the number of surface structures are different at each case, we only presented surface charge density over 5 surface structure since the remaining will be identical. EDL overlap starts at the deep of the valley and decreases towards the top region of the roughness geometry. Absolute value of surface charge in the valleys are lower and on the hills are higher due to EDL overlap and curvature effects, respectively. When the roughness diameter is smaller than the EDL thickness, the overlap results in decrease of absolute surface charge. Hence, the absolute value of average of local surface charge distribution yield lower values than the flat SNP surface charge at the corresponding particle diameter and temperature. This difference decreases as the roughness diameter increase. Basically, both EDL overlap and curvature effects decrease with an increase in the size of roughness structures. This also shows itself as decreasing fluctuations in local variation of nanoparticle surface charge. The increase of temperature increases the absolute charge values as well as the difference between the hills and valleys of surface roughness. At this point, we can simply state that temperature influence is stronger on rough SNPs and increases by the decrease of roughness diameter. 


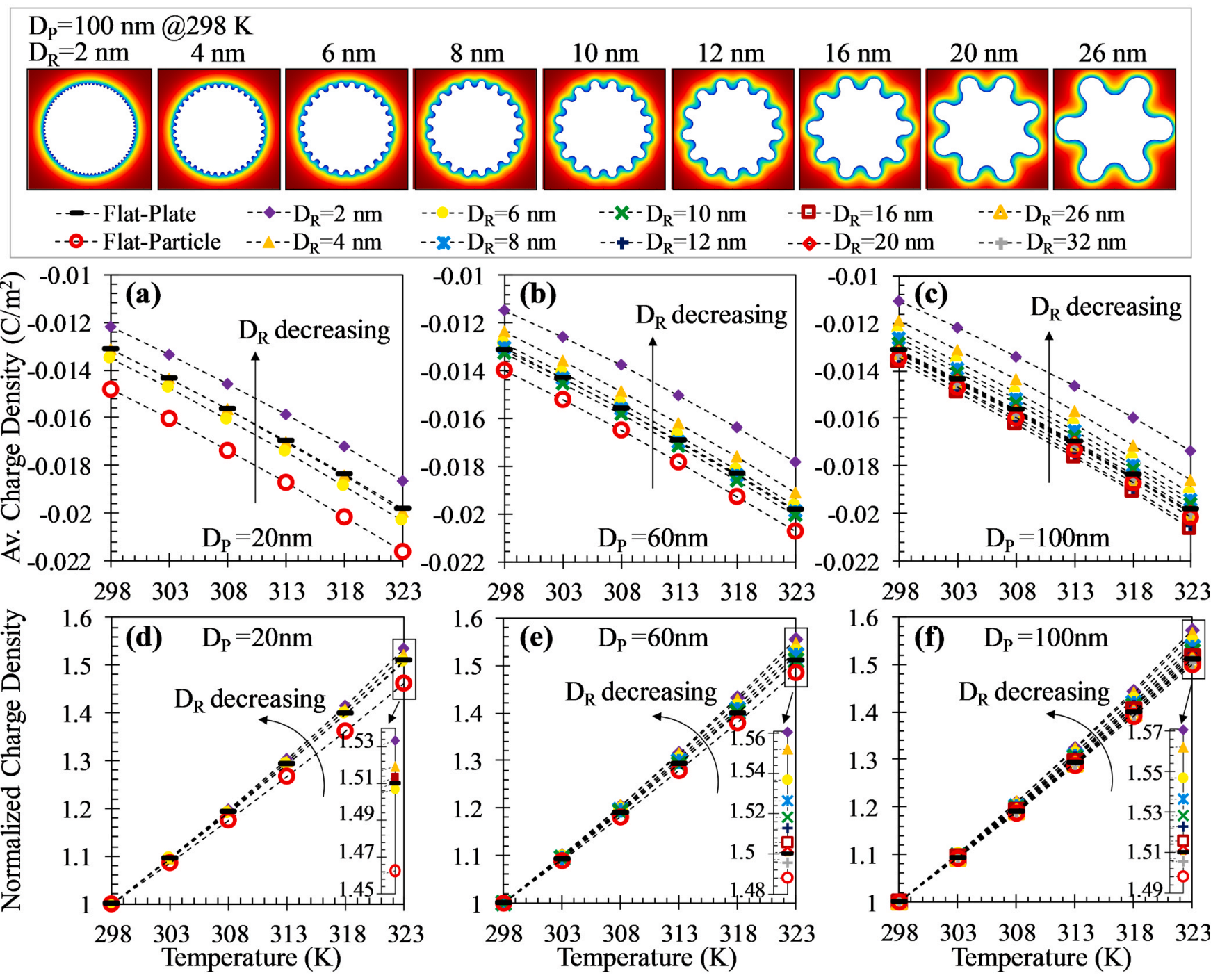

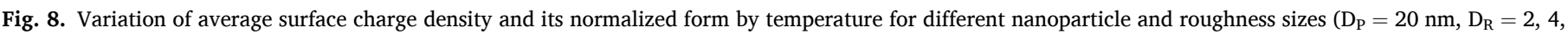

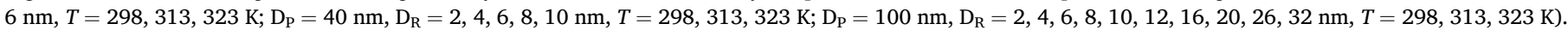

The average of surface charge densities around the rough nanoparticles are presented as a function of temperature in Fig. 8. We also presented surface charge calculations of $\mathrm{PB}$ theory at the corresponding ionic conditions. We should mention again that these $\mathrm{PB}$ calculations assume a flat surface with an uninterrupted EDL, i.e. no curvature effects or no EDL overlap, but consider similar temperature dependent property models including the CR with $K_{A}=K_{A}(T)$. Variation of surface roughness by roughness diameter is illustrated on top of the figure for $100 \mathrm{~nm}$ SNP at $298 \mathrm{~K}$ as the roughness diameter is varied between $2 \mathrm{~nm}$ and $32 \mathrm{~nm}$. Temperature effect on the average surface charge of $D_{P}=20,40$ and $100 \mathrm{~nm}$ at various surface roughness are plotted in Fig. 8(a), (b) and (c). At a constant particle diameter, absolute value of the average surface charge decreases with an increase in surface roughness. While the PB calculated surface charge for a flat plate is a constant value for the given ionic condition and temperature, surface charge of SNP shows variation by particle size and roughness level. Simply, curvature effects alone develop higher absolute charges than a flat plate prediction by the decrease of particle size of a flat particle; but including surface roughness creates EDL overlap which decreases the absolute surface charge by decreasing roughness diameter. Surface structures equal and smaller than $2 \mathrm{~nm}$ requires definition of "effective boundary for electrokinetic interactions" and consideration of atomistic details, similar to earlier studies [45].

The average surface charge densities normalized with charge density values at $298 \mathrm{~K}$ for each particle and roughness diameters are given in Fig. 8(d), (e) and (f). We present the temperature influence on each case by calculating the variation in surface charge compare to its charge value at $298 \mathrm{~K}$. Temperature effects appeared to be increasing with a decrease in surface roughness. Variation of normalized surface charge by temperature remains mostly linear, but the rate of increase advances by decreasing surface roughness size. While $1 \mathrm{~K}$ increase in temperature create $2 \%$ increase in surface charge of a $100 \mathrm{~nm}$ flat SNP, a $100 \mathrm{~nm}$ SNP with $2 \mathrm{~nm}$ roughness diameter undergoes a $2.3 \%$ charge change per $1 \mathrm{~K}$. Hence, an increase of temperature eventually decreases the negative influence (decreasing the absolute surface charge) of surface roughness. So, lower roughness cases develop higher temperature dependent variation. Next, we studied temperature effects on rough SNPs under varying particle size with constant roughness diameter. Fig. 9 presents electric potential contours of $D_{P}=100,60,40$ and $20 \mathrm{~nm}$ with $D_{R}$ $=6 \mathrm{~nm}$. Results show that absolute surface charge increases with a decrease in SNP diameter. As the roughness diameters of these cases are same, EDL overlaps remain mostly the same so that increase of absolute charge is mostly due to increasing curvature effects. Detailed discussions on curvature effects on size dependent surface charging of SNPs can be 

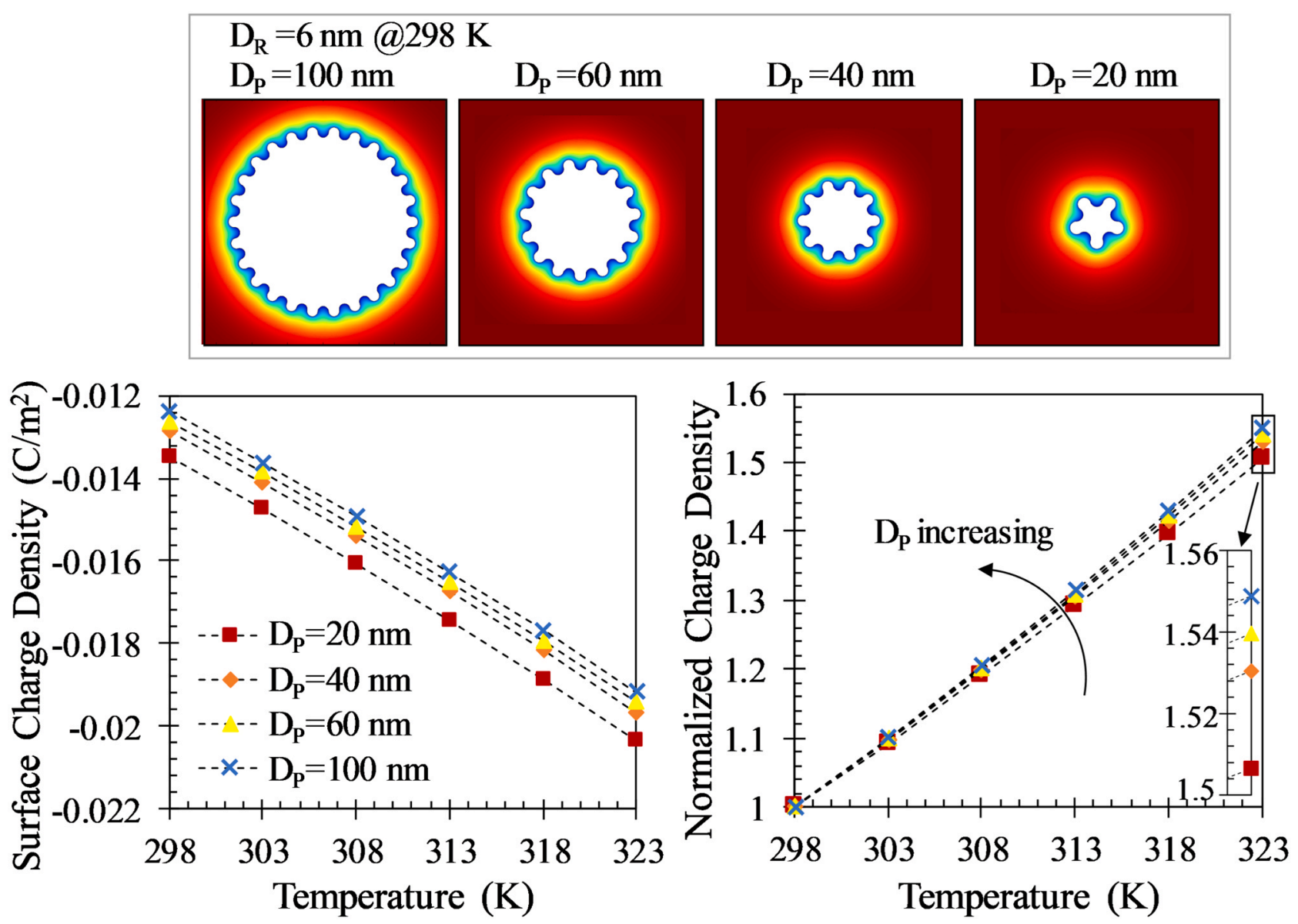

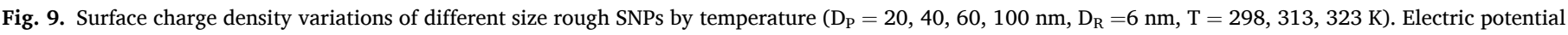
contours are at $298 \mathrm{~K}$.

found in our earlier study [45]. Next, we normalized surface charges with their corresponding values at $298 \mathrm{~K}$. Decrease of particle size decreases the influence of temperature. Hence, the positive influence (increasing the absolute surface charge) of curvature effects lessens with a temperature increase.

We summarized the temperature influence on surface charging of different size rough SNPs as a function of roughness diameter in Fig. 10 (a). The percentage increase in surface charge per $1 \mathrm{~K}$ decreases by increasing roughness size and/or decreasing particle diameter. Hence, temperature influence shows variation by particle and roughness size. In our earlier study [45], we presented that an "electrokinetic similarity" between different systems of various size and roughness develops based on the non-dimensional groups of $\lambda / D_{P}$ and $\lambda / D_{R}$ for the characterization of curvature and EDL overlap. These effects have opposite influence at different levels. We successfully obtained a universal behavior in temperature dependent surface charge variation as a function of the
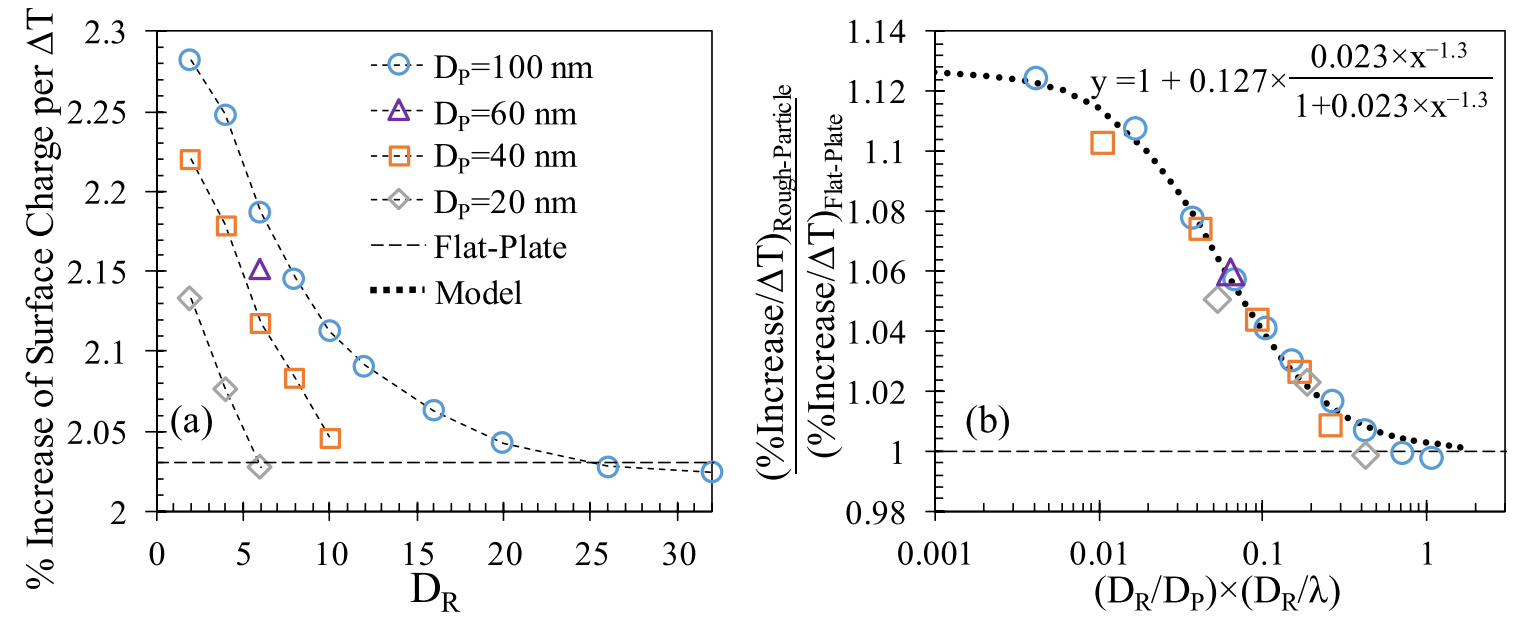

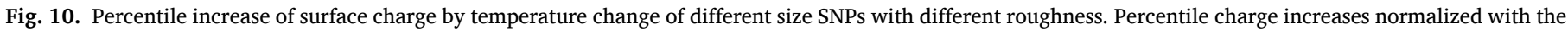
percentile change calculated by flat plate theory. 


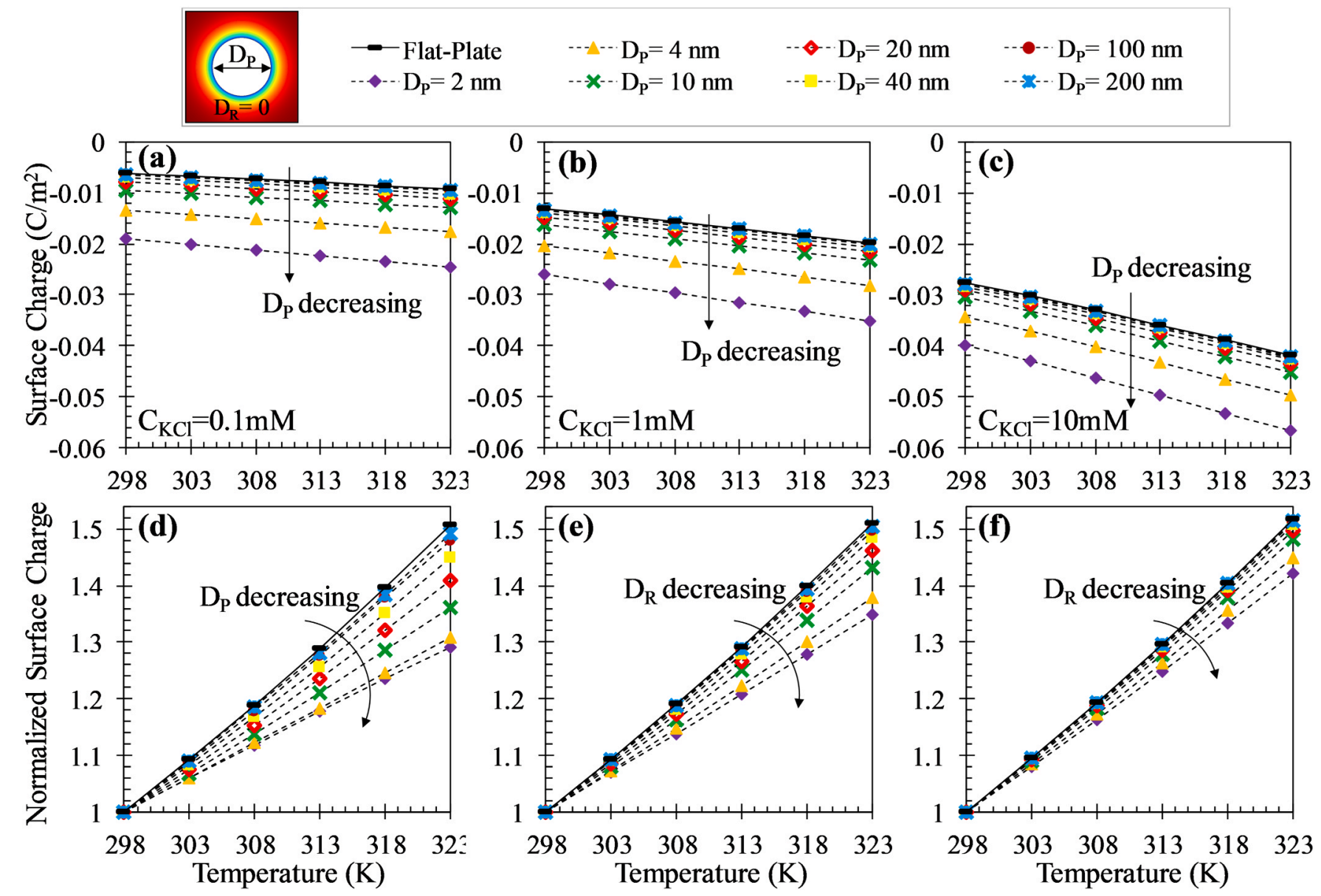

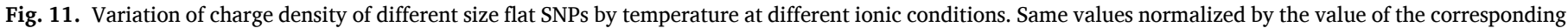
case at $298 \mathrm{~K}$.

non-dimensional set of $\left(D_{R} / D_{P}\right) \times\left(\lambda / D_{R}\right)$ in Fig. 10(b). Based on the results, the increment of SNP surface charge by temperature differs from the existing PB theory when $\left(D_{R} / D_{P}\right) \times\left(\lambda / D_{R}\right)$ becomes less than $\sim 5$. This is basically due to change in curvature and EDL overlap effects by the change of temperature.

In an attempt to characterize the observed behavior, we applied curve fitting on the data given in Fig. 10 (b). The trigonometric function below provides a good description for the temperature dependence of rough particle surface charging compare to the temperature dependence of a flat surface as a function of $\left(D_{R} / D_{P}\right) \times\left(\lambda / D_{R}\right)$.
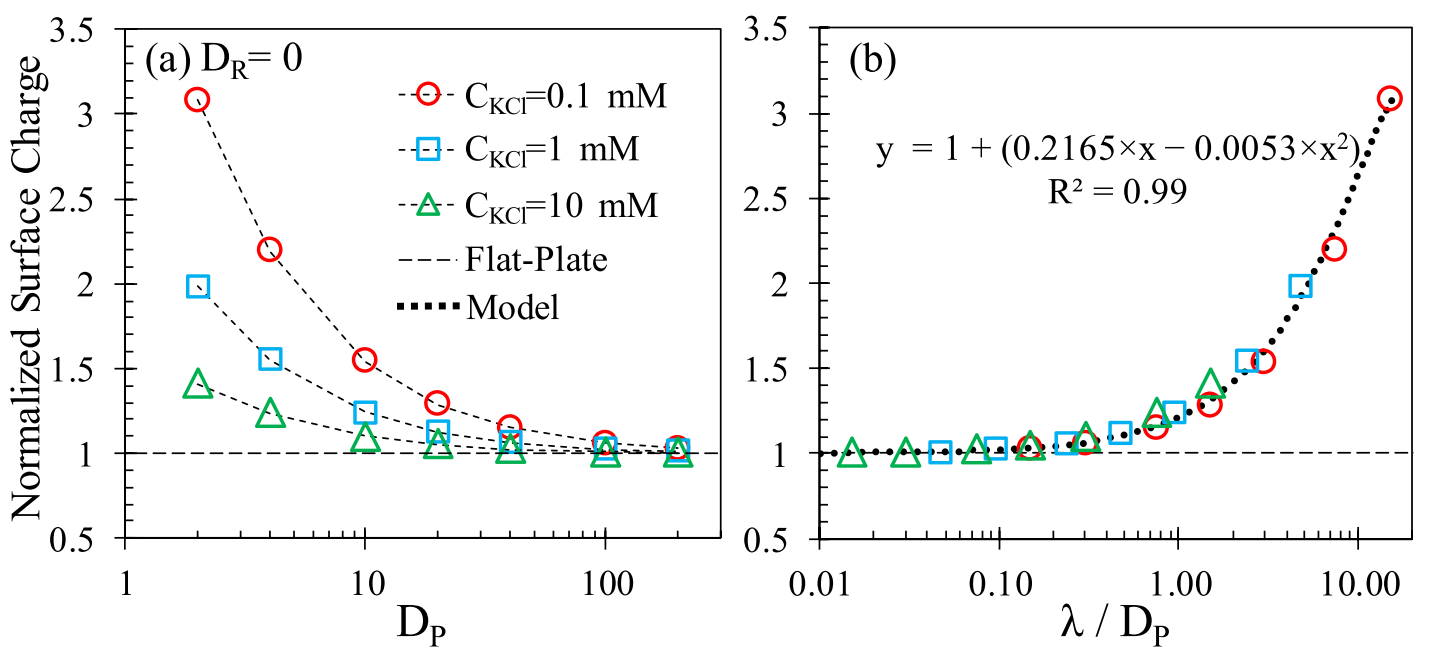

Fig. 12. Surface charge of flat SNPs at different particle diameters and salt concentrations normalized with the flat plate theory calculations for the corresponding ionic conditions. 

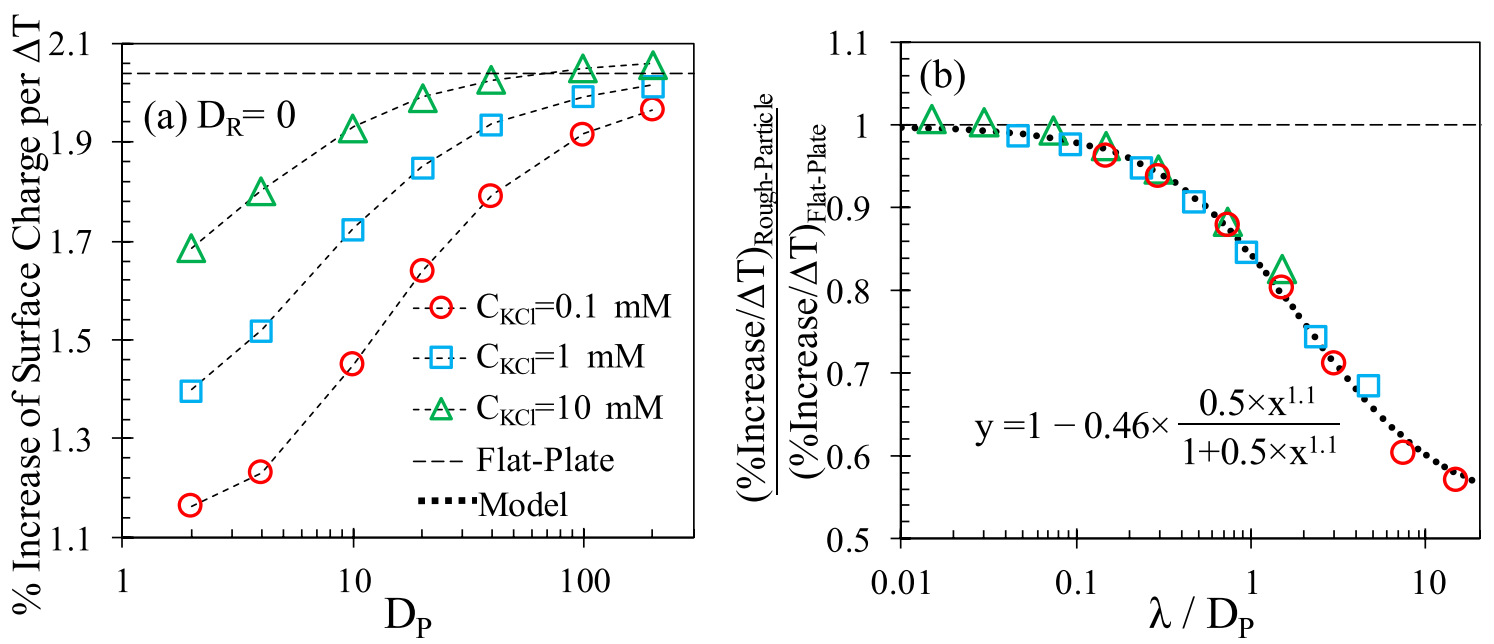

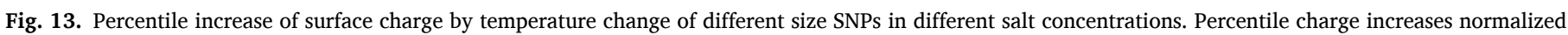
with the percentile change calculated by flat plate theory.

$$
\frac{(\% \text { increase } / \Delta T)_{\text {Rough-Particle }}}{(\% \text { increase } / \Delta T)_{\text {Flat-Plate }}}=1+0.127 \frac{0.023\left(\frac{D_{R}^{2}}{D_{P} \times \lambda}\right)^{-1.3}}{1+0.023\left(\frac{D_{R}^{2}}{D_{P} \times \lambda}\right)^{-1.3}}
$$

Eq. (24) converges to value of unity when the $\left(D_{R} / D_{P}\right) \times\left(\lambda / D_{R}\right) \rightarrow$ 0 representing temperature dependence reaches to behavior of a flat surface. To predict surface charge of a rough SNP at a given temperature, we extended our earlier developed phenomenological model as,

$$
\begin{aligned}
\frac{\sigma_{\text {Rough-Particle-Ave }}(T)}{\sigma_{\text {Flat-Plate }}(T)}= & \left(1-A \times \exp \left(-B \times\left(\frac{D_{R}^{2}}{D_{P} \times \lambda}\right)\right)\right) \\
& \times\left(1+\frac{(T-298)}{100} \times \frac{0.00292\left(\frac{D_{R}^{2}}{D_{P} \times \lambda}\right)^{-1.3}}{1+0.023\left(\frac{D_{R}^{2}}{D_{P} \times \lambda}\right)^{-1.3}}\right)
\end{aligned}
$$

where $\mathrm{A}$ and $\mathrm{B}$ are constants varying by $\mathrm{pH}$.

$$
\begin{aligned}
& A=0.00167 \times p H^{4}-0.0369 \times p H^{3}+0.279 \times p H^{2}-0.813 \times p H+0.8 \\
& B=0.409 \times p H^{3}-4.55 \times p H^{2}+17.12 \times p H-18
\end{aligned}
$$

In Eq. (25), the first group of terms in parenthesis is the phenomenological model developed in our earlier study [45] to described the exponential decrease of rough SNP surface charge by the decrease of $\left(\mathrm{D}_{\mathrm{R}}^{2} /\left(\lambda \times \mathrm{D}_{\mathrm{P}}\right)\right)$ parameter with respect to analytical flat plate calculations given in Eq. (23). However, this model was devised at $298 \mathrm{~K}$. If the temperature dependence of a SNP was similar to flat plate case, this model could provide rough SNP surface charge from the value of flat plate at the corresponding temperature predicted by Eq. (23). But, current results show that variation of SNP surface charge by temperature is different that theory. So, the second, parenthetical group of terms includes this deviation in the calculations. Simply, if the temperature is at $298 \mathrm{~K}$, the second part becomes 0 and the remaining is the earlier developed model. But, if there is a finite temperature increase from $298 \mathrm{~K}$, the second part considers higher response of SNP surface charge variation per Kelvin temperature change.

As a next step, we focused on the temperature dependent surface charging of flat SNPs as a function of particle diameter and ionic concentration. In Fig. 11(a), (b), and (c), we present variation of surface charge of SNPs with $\mathrm{D}_{\mathrm{P}}=2,4,10,20,40,100 \mathrm{~nm}$ at $\mathrm{C}_{\mathrm{KCl}}=0.1,1$, and $10 \mathrm{mM}$ electrolytes with $\mathrm{pH}=7$ as a function of temperature. We also added surface charge calculations of PB theory (Eq. (23)) for each temperature value at the corresponding ionic conditions. The PB theory assuming an uninterrupted EDL over a flat plate also includes the CR with similar temperature dependent models. For such a case, surface charge of SNPs with $D_{P} \geq 100 \mathrm{~nm}$ and at flat surface condition agrees with PB solution. However, a decrease in nanoparticle size less than $100 \mathrm{~nm}$ yields an increase of absolute surface charge; charge on a $2 \mathrm{~nm}$ SNP almost 2 times the calculations of flat plate theory ( $\sigma_{\text {Flat-Particle-100nm }}$ $=-0.01348 \mathrm{C} / \mathrm{m}^{2}$ while $\sigma_{\text {Flat-Particle-2nm }}=-0.02608 \mathrm{C} / \mathrm{m}^{2}$ at $298 \mathrm{~K}$ ). This is due to curvature effects and details can be found in our earlier study [45]. For all particle sizes, increase of temperature creates substantial increase of absolute surface charge values; $25 \mathrm{~K}$ increase develops more than $50 \%$ charge increase. Change of surface charge is almost linear so that we can simply conclude that charge change is around $2 \% 1 / \mathrm{K}$ for flat plate $\mathrm{PB}$ solution. In order to compare the influence of temperature on different size particles, we normalized the surface charge values with the charge of corresponding particle size at $298 \mathrm{~K}$ in Fig. 11(d), (e), and (f). Results show that a decrease in particle size eventually decreases the effect of temperature change. While variation by temperature remains linear, the slope of these curves decreases by decreasing the particle diameter. For example, change of charge by temperature is $2 \% 1 / \mathrm{K}$ for $100 \mathrm{~nm}$ SNP while it decreases to $1.4 \%$ per $\mathrm{K}$ for $2 \mathrm{~nm}$ SNP. This eventually decreases the difference between the surface charge of $100 \mathrm{~nm}$ and $2 \mathrm{~nm}$ SNPs.

In Fig. 12, we present surface charge variation of a flat SNP by its particle diameter and ionic condition at $298 \mathrm{~K}$. Fig. 12(a) shows that SNP surface charge drastically increases by the decrease of particle diameter; but this increase is different at different salt levels. This is due to the change of EDL thickness by the change in salt concentration. We obtained a universal behavior when we analyzed the results as a function of $\lambda / D_{\mathrm{P}}$ in Fig. 12(b). Simply, SNP surface charge increases by the increase of $\lambda / D_{\mathrm{P}}$, while SNP charge converges back to flat surface values for $\lambda / D_{P}<0.2$. This is an outcome similar to our earlier study describing surface charge of flat SNPs as a function of $\lambda / D_{P}$ [45]. We observed that the second order polynomial function given in Eq. (27) describes this behavior very well.

$\frac{\sigma_{\text {Flat-Particle } @ 298 K}}{\sigma_{\text {Flat-Plate } 298 K}}=1+\left(0.2165 \times\left(\frac{\lambda}{D_{P}}\right)-0.0053 \times\left(\frac{\lambda}{D_{P}}\right)^{2}\right)$

Next, we studied percentage increase of flat particle's surface charge by per $1 \mathrm{~K}$ increase in temperature as a function of particle diameter in Fig. 13(a). Surface charge variation by temperature lessens with the decrease in particle diameter and/or salt concentration. These cases develop different level of curvature effects. As we described, the curvature effects develop as a function of the ratio of the EDL thickness to particle diameter. So, temperature influence differs at cases with 

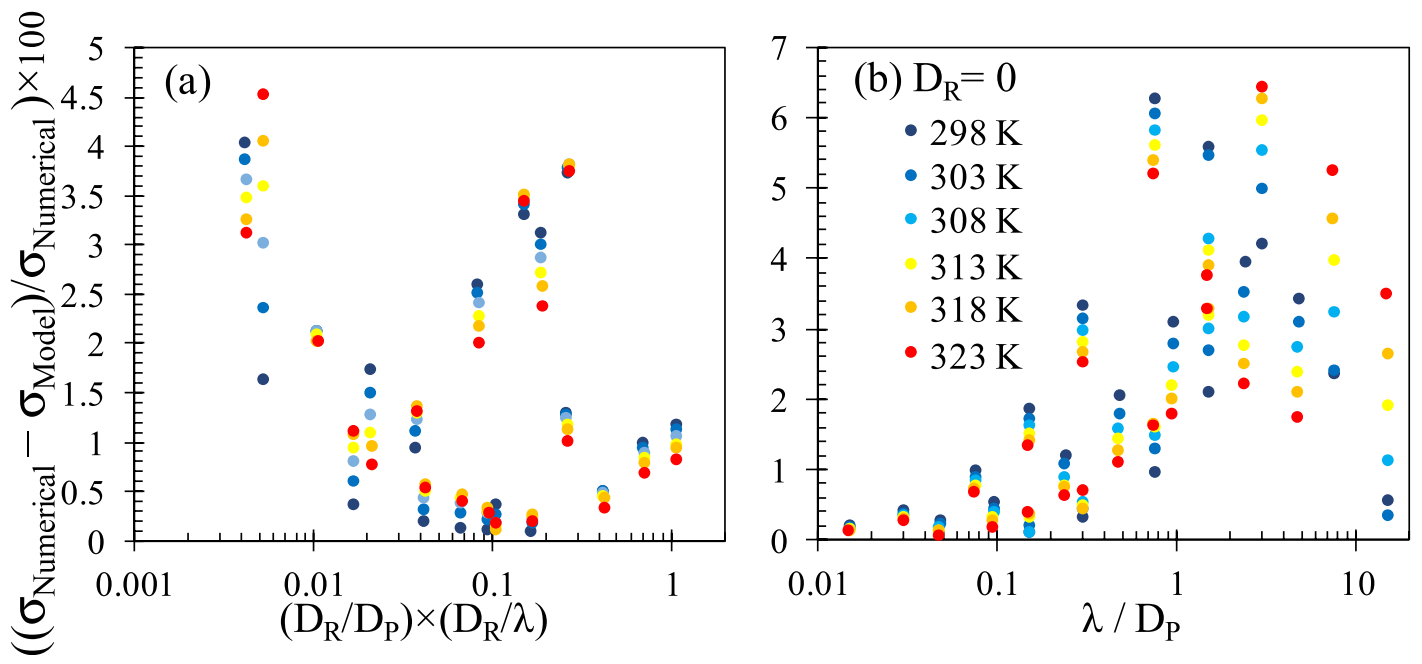

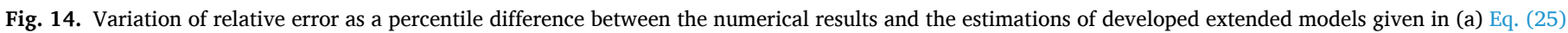
and (b) Eq. (29).

different curvature effects. Basically, temperature rise not only enhances the ionic concentration and reaction constants on the surface, but it also changes the curvature effects. In Fig. 13(b), we present deviation of the percentile surface charge increase of SNP from the flat theory predictions as a function of $\lambda / D_{\mathrm{p}}$. We obtained a "electrokinetic similarity" for curvature effects of different systems as a function of the nondimensional groups of $\lambda / D_{p}$. Results show that percentage increase of flat SNP's surface charge per temperature change is similar to PB calculated values for a flat plate when $\lambda / D_{P}>0.1$. On the other hand, the temperature influence decreases substantially by the decrease of $\lambda / \mathrm{D}_{\mathrm{P}}$ below the value of 0.1 . Next, we applied a trigonometric function similar to one used earlier. We were able to obtain a very successful description for the universal behavior obtained in the temperature dependence of different size particles immersed in electrolytes of different salt concentrations in Eq. (28). Eq. (28) converges to value of unity when the $\left(\lambda / D_{P}\right) \rightarrow 0$ representing temperature dependence reaches to behavior of a flat surface. Conversely, flat SNP surface charging deviates from flat plate theory up to $50 \%$ decrease in temperature dependent surface charge increase.

$$
\frac{(\% \text { increase } / \Delta T)_{\text {Flat-Particle }}}{(\% \text { increase } / \Delta T)_{\text {Flat }- \text { Plate }}}=1-0.46 \frac{0.5\left(\frac{\lambda}{D_{P}}\right)^{1.1}}{1+0.5\left(\frac{\lambda}{D_{P}}\right)^{1.1}}
$$

Next, we combined the developed Eqs. (27) and (28) to describe the surface charge of a certain flat SNP at a given temperature as,

$$
\begin{aligned}
\frac{\sigma_{\text {Flat-Particle }}(T)}{\sigma_{\text {Flat-Plate }}(T)}= & \left(1+0.2165 \times\left(\frac{\lambda}{D_{P}}\right)-0.0053 \times\left(\frac{\lambda}{D_{P}}\right)^{2}\right) \\
& \times\left(1-\frac{(T-298)}{100} \times \frac{0.23\left(\frac{\lambda}{D_{P}}\right)^{1.1}}{1+0.5\left(\frac{\lambda}{D_{P}}\right)^{1.1}}\right)
\end{aligned}
$$

Eq. (29) simply extents existing theory given in Eq. (23) to include particle size effects as a phenomenological model for SNP surface charging. As the final step, we compared the surface charge estimations of Eqs. (25) and (29) with the numerical calculations in Fig. 14. Any roughness diameter smaller than $2 \mathrm{~nm}$, the effective surface charge estimation moves from Eq. (25) to Eq. (29). We described this behavior in an earlier study. Fig. 14(a) presents the percentile errors of Eq. (25) while Fig. 14(b) gives the errors of Eq. (29). For both model, error values mostly remain lower than $3 \%$ and $4 \%$ while the highest errors were around $4 \%$ and $6 \%$ for only few cases.

\section{Conclusions}

Silica nanoparticles (SNP) at different particle sizes with different surface areas are used in numerous micro/nanofluidic applications, while their surface charge properties plays a major role in their function. In many of these applications, SNPs also undergo some temperature variation. SNP surface charge deviates from theoretical predictions due to curvature and EDL overlap effects. Furthermore, this differentiation from theory also changes by temperature. For such a case, surface charge behavior of SNPs of various size and surface conditions are studied at different temperatures. Surface physiochemistry is properly calculated by considering temperature dependent variation in permittivity, ionic mobility, and equilibrium constants of the surface reactions coupled with a CR model. Results show that the absolute value of SNP surface charge undergoes a substantial increase by the increase of temperature while this change develops as a strong function of particle diameter and surface condition. When the particle and roughness size becomes comparable with EDL thickness, curvature effect and EDL overlap develop which creates divergence from the known PB based theories. These two effects create opposite influence on temperature dependence of SNP's surface charging. While the decrease of particle size lessens the temperature influence, the decrease of roughness size enhances the temperature dependence. For such a case, we systematically studied surface charge of SNPs of different sizes at various level of roughness defined by roughness diameter parameter up to the flat surface condition. Specifically, temperature dependence increases with decreasing roughness diameter; surface charge variation by temperature of different size particles with different roughness diameters increases by decreasing roughness diameter while it shows a universal behavior as a function of $\left(D_{R} / D_{P}\right) \times\left(\lambda / D_{R}\right)$. In our earlier study, we present "electrokinetic similarity" between different systems of various size and roughness based on the non-dimensional groups of $\left(D_{R} / D_{P}\right) \times\left(\lambda / D_{R}\right)$ as a function of which we developed a phenomenological model for the characterization of curvature and EDL overlap effects on rough SNP surface charging [45]. A similar conclusion also appeared in temperature influence. For such a case, we extend the earlier model to include temperature dependence. By increasing roughness diameter, the influence of temperature converges back to $\mathrm{PB}$ prediction for $\left(\mathrm{D}_{R} / \mathrm{D}_{\mathrm{P}}\right) \times\left(\lambda / \mathrm{D}_{\mathrm{R}}\right)>5$. Conversely, decrease of roughness size reaches to a flat surface particle. In this transition, the boundary effective in electrokinetic interactions changes from particle surface to an arc defined as a function of an angle $\theta$ representing the point where the concave circular arc of the hill meets with the convex arc of the valley. Detailed discussions on the "effective boundary for electrokinetic interactions" can be found in our earlier 
study. In flat SNP condition, curvature effects dominate and create significant divergence from PB theory without the opposite and balancing effect of EDL overlap. Surface charge increases up to 3 times of flat plate theory calculations, but this difference decreases with an increase in temperature. Decrease of particle size creates up to $40 \%$ decrease in the temperature dependence compare to $\mathrm{PB}$ predictions. As a function of $\lambda / \mathrm{D}_{\mathrm{P}}$, we obtain an electrokinetic similarity between different size flat SNPs immersed in electrolytes of different salt concentrations. Results show that curvature effects become negligible for $\lambda / D_{P}<0.1$. First, we developed a phenomenological model for surface charge behavior of flat SNP at $298 \mathrm{~K}$, and later, for its temperature dependence, as an extension to PB theory. This study shows that temperature change not only affects the surface chemistry, but also creates a substantial change in the recently described scale effects of nanoparticles, namely curvature effects and EDL overlap on the surface. As a result, temperature variation creates drastically different effects on SNPs as a function of particle size and roughness level. The current findings are very important for the characterization of SNPs through applications consisting temperature variations. Results can also be used for adjusting/tuning the surface charge of SNPs to control the related physics by tuning the temperature.

\section{Declaration of Competing Interest}

The authors declare that they have no known competing financial interests or personal relationships that could have appeared to influence the work reported in this paper.

\section{Acknowledgments}

This work was supported by the Scientific and Technological Research Council of Turkey (TUBITAK) under the Grant Number 118M710. Authors also would like to thank Center for Scientific Computation at Southern Methodist University. This work was also supported by the BAGEP Award of the Science Academy.

\section{References}

[1] M. Liong, J. Lu, M. Kovochich, T. Xia, S.G. Ruehm, A.E. Nel, F. Tamanoi, J.I. Zink, Multifunctional inorganic nanoparticles for imaging, targeting, and drug delivery, ACS Nano 2 (2008) 889-896, https://doi.org/10.1021/nn800072t.

[2] J.E. Lee, N. Lee, T. Kim, J. Kim, T. Hyeon, Multifunctional mesoporous silica nanocomposite nanoparticles for theranostic applications, Acc. Chem. Res. 44 (2011) 893-902, https://doi.org/10.1021/ar2000259.

[3] I.I. Slowing, B.G. Trewyn, S. Giri, V.S.Y. Lin, Erratum: Borderud SP, Li Y, Burkhalter JE, Sheffer CE and Ostroff JS. Electronic cigarette use among patients with cancer: characteristics of electronic cigarette users and their smoking cessation outcomes, Cancer 121 (2015) 800, https://doi.org/10.1002/ adfm. 200601191.

[4] A. Popat, S.B. Hartono, F. Stahr, J. Liu, S.Z. Qiao, G. Qing Max Lu, Mesoporous silica nanoparticles for bioadsorption, enzyme immobilisation, and delivery carriers, Nanoscale 3 (2011) 2801-2818, https://doi.org/10.1039/c1nr10224a.

[5] K. Ariga, Q. Ji, T. Mori, M. Naito, Y. Yamauchi, H. Abe, J.P. Hill, Enzyme nanoarchitectonics: organization and device application, Chem. Soc. Rev. 42 (2013) 6322-6345, https://doi.org/10.1039/c2cs35475f.

[6] Z. Li, J.C. Barnes, A. Bosoy, J.F. Stoddart, J.I. Zink, Mesoporous silica nanoparticles in biomedical applications, Chem. Soc. Rev. 41 (2012) 2590-2605, https://doi org/10.1039/c1cs15246g.

[7] J.L. Vivero-Escoto, I.I. Slowing, V.S.Y. Lin, B.G. Trewyn, Mesoporous silica nanoparticles for intracellular controlled drug delivery, Small 6 (2010) 1952-1967, https://doi.org/10.1002/smll.200901789.

[8] P. Yang, S. Gai, J. Lin, Functionalized mesoporous silica materials for controlled drug delivery, Chem. Soc. Rev. 41 (2012) 3679-3698, https://doi.org/10.1039/ c2 2 15308d.

[9] M. Zhang, L.H. Yeh, S. Qian, J.P. Hsu, S.W. Joo, DNA electrokinetic translocation through a nanopore: local permittivity environment effect, J. Phys. Chem. C 116 (2012) 4793-4801.

[10] Y. Ai, J. Liu, B. Zhang, S. Qian, Field effect regulation of DNA translocation through a nanopore, Anal. Chem. 82 (2010) 8217-8225, https://doi.org/10.1021/ ac101628e.

[11] X. Li, Q.R. Xie, J. Zhang, W. Xia, H. Gu, The packaging of siRNA within the mesoporous structure of silica nanoparticles, Biomaterials 32 (2011) 9546-9556, https://doi.org/10.1016/j.biomaterials.2011.08.068.

[12] C.K. Wang, H.K. Tsao, Temperature-insensitive electrokinetic behavior in capillary zone electrophoresis, J. Phys. Chem. B 108 (2004) 17685-17693.
[13] G.Y. Tang, C. Yang, H.Q. Gong, J.C. Chai, Y.C. Lam, Numerical simulation of Joule heating effect on sample band transport in capillary electrophoresis, Anal. Chim. Acta 561 (2006) 138-149.

[14] C.J. Evenhuis, V. Hruska, R.M. Guijt, M. Macka, B. Gas, P.J. Marriott, P.R. Haddad, Reliable electrophoretic mobilities free from Joule heating effects using CE, Electrophoresis 28 (2007) 3759-3766.

[15] M. Taghipoor, A. Bertsch, P. Renaud, Thermal control of ionic transport and fluid flow in nanofluidic channels, Nanoscale 7 (2015) 18799-18804.

[16] S. Tseng, Y.M. Li, C.Y. Lin, J.P. Hsu, Salinity gradient power: influences of temperature and nanopore size, Nanoscale 8 (2016) 2350-2357.

[17] M. Taghipoor, A. Bertsch, P. Renaud, Temperature sensitivity of nanochannel electrical conductance, ACS Nano 9 (2015) 4563-4571.

[18] J.P. Hsu, Y.H. Tai, L.H. Yeh, S. Tseng, Importance of temperature effect on the electrophoretic behavior of charge-regulated particles, Langmuir 28 (2012) 1013-1019.

[19] J. Hwang, T. Sekimoto, W.L. Hsu, S. Kataoka, A. Endo, H. Daiguji, Thermal dependence of nanofluidic energy conversion by reverse electrodialysis, Nanoscale 9 (2017) 12068-12076.

[20] B.W. Ninham, V.A. Parsegian, Electrostatic potential between surfaces bearing ionizable groups in ionic equilibrium with physiologic saline solution, J. Theor. Biol. 31 (1971) 405-428, https://doi.org/10.1016/0022-5193(71)90019-1.

[21] V.E. Shubin, P. Kékicheff, Electrical double layer structure revisited via a surface force apparatus: mica interfaces in lithium nitrate solutions, J. Colloid Interface Sci. 155 (1993) 108-123, https://doi.org/10.1006/jcis.1993.1016.

[22] J.P. Chapel, Electrolyte species dependent hydration forces between silica surfaces, Langmuir 10 (1994) 4237-4243, https://doi.org/10.1021/la00023a053.

[23] M. Dishon, O. Zohar, U. Sivan, From repulsion to attraction and back to repulsion: The effect of $\mathrm{NaCl}, \mathrm{KCl}$, and $\mathrm{CsCl}$ on the force between silica surfaces in aqueous solution, Langmuir 25 (2009) 2831-2836, https://doi.org/10.1021/la803022b.

[24] I. Popa, P. Sinha, M. Finessi, P. Maroni, G. Papastavrou, M. Borkovec, Importance of charge regulation in attractive double-layer forces between dissimilar surfaces, Phys. Rev. Lett. 104 (2010), 228301, https://doi.org/10.1103/ PhysRevLett.104.228301.

[25] R. Nosrati, M. Hadigol, M. Raisee, A. Nourbakhsh, Uncertainty and bias in contrast concentration measurements using spoiled gradient echo pulse sequences, Phys. Med. Biol. 53 (2008) 2345-2373, https://doi.org/10.1166/jctn.2012.2643.

[26] T. Sen, M. Barisik, Size dependent surface charge properties of silica nanochannels: double layer overlap and inlet/outlet effects, Phys. Chem. Chem. Phys. 20 (2018) 16719-16728, https://doi.org/10.1039/c8cp01906a.

[27] K. Da Huang, R.J. Yang, Electrokinetic behaviour of overlapped electric double layers in nanofluidic channels, Nanotechnology 18 (2007), 115701.

[28] L.-H.H. Yeh, S. Xue, S.W. Joo, S. Qian, J.-P.P. Hsu, Field effect control of surface charge property and electroosmotic flow in nanofluidics, J. Phys. Chem. C 116 (2012) 4209-4216.

[29] C. Hughes, L.H. Yeh, S. Qian, Tuning ion transport and selectivity by a salt gradient in a charged nanopore, Anal. Chem. 86 (2014) 2681-2686, https://doi.org/ 10.1021/jp402018u.

[30] L.H. Yeh, M. Zhang, S. Qian, Ion transport in a pH-regulated nanopore, Anal. Chem. 85 (2013) 7527-7534, https://doi.org/10.1021/ac401536g.

[31] L.H. Yeh, C. Hughes, Z. Zeng, S. Qian, Tuning ion transport and selectivity by a salt gradient in a charged nanopore, Anal. Chem. 86 (2014) 2681-2686, https://doi. org/10.1021/ac4040136.

[32] Y. Ai, M. Zhang, S.W. Joo, M.A. Cheney, S. Qian, Effects of electroosmotic flow on ionic current rectification in conical nanopores, J. Phys. Chem. C 114 (2010) 3883-3890, https://doi.org/10.1021/jp911773m.

[33] Z. Zeng, L.H. Yeh, M. Zhang, S. Qian, Ion transport and selectivity in biomimetic nanopores with pH-tunable zwitterionic polyelectrolyte brushes, Nanoscale 7 (2015) 17020-17029, https://doi.org/10.1039/c5nr05828g.

[34] T. Sen, M. Barisik, Internal surface electric charge characterization of mesoporous silica, Sci. Rep. 9 (2019) 137, https://doi.org/10.1038/s41598-018-36487-w.

[35] T. Sen, M. Barisik, Pore connectivity effects on the internal surface electric charge of mesoporous silica, Colloid Polym. Sci. 297 (2019) 1365-1373, https://doi.org/ 10.1007/s00396-019-04555-w.

[36] M. Barisik, S. Atalay, A. Beskok, S. Qian, Size dependent surface charge properties of silica nanoparticles, J. Phys. Chem. C. 118 (2014) 1836-1842.

[37] S. Atalay, M. Barisik, A. Beskok, S. Qian, Surface charge of a nanoparticle interacting with a flat substrate, J. Phys. Chem. C 118 (2014) 10927-10935, https://doi.org/10.1021/jp5023554.

[38] R.J. Hill, Corona charge regulation in nanoparticle electrophoresis, Proc. R. Soc. A 471 (2015), 20150522, https://doi.org/10.1098/rspa.2015.0522.

[39] Y.R. Shi, M.P. Ye, L.C. Du, Y.X. Weng, Experimental determination of particle sizedependent surface charge density for silica nanospheres, J. Phys. Chem. C 122 (2018) 23764-23771, https://doi.org/10.1021/acs.jpcc.8b07566.

[40] M. Kobayashi, F. Juillerat, P. Galletto, P. Bowen, M. Borkovec, Aggregation and charging of colloidal silica particles: effect of particle size, Langmuir 21 (2005) 5761-5769, https://doi.org/10.1021/la046829z.

[41] F. Borghi, V. Vyas, A. Podestà, P. Milani, Nanoscale roughness and morphology affect the isoelectric point of titania surfaces, PLoS One 8 (2013) 68655, https:// doi.org/10.1371/journal.pone.0068655.

[42] J.F.L. Duval, F.A.M. Leermakers, H.P. Van Leeuwen, Electrostatic interactions between double layers: influence of surface roughness, regulation, and chemical heterogeneities, Langmuir 20 (2004) 5052-5065, https://doi.org/10.1021/ la030404f.

[43] X. Yang, G. Zhang, The effect of an electrical double layer on the voltammetric performance of nanoscale interdigitated electrodes: a simulation study, 
Nanotechnology 19 (2008), 465504, https://doi.org/10.1088/0957-4484/19/46/ 465504.

[44] H.G. Ozcelik, M. Barisik, Electric charge of nanopatterned silica surfaces, Phys. Chem. Chem. Phys. 21 (2019) 7576-7587, https://doi.org/10.1039/c9cp00706g.

[45] B.O. Alan, M. Barisik, H.G. Ozcelik, Roughness effects on the surface charge properties of silica nanoparticles, J. Phys. Chem. C 124 (2020) 7274-7286, https:// doi.org/10.1021/acs.jpcc.0c00120.

[46] Y. Ma, L.H. Yeh, S. Qian, PH-regulated ionic conductance in a nanopore, Electrochem. Commun. 43 (2014) 91-94, https://doi.org/10.1016/j. elecom.2014.03.024.

[47] L.H. Yeh, Y.H. Tai, N. Wang, J.P. Hsu, S. Qian, Electrokinetics of pH-regulated zwitterionic polyelectrolyte nanoparticles, Nanoscale 4 (2012) 7575-7584, https://doi.org/10.1039/c2nr32277c.

[48] L.H. Yeh, J.P. Hsu, S. Qian, S. Tseng, Counterion condensation in pH-regulated polyelectrolytes, Electrochem. Commun. 19 (2012) 97-100, https://doi.org/ 10.1016/j.elecom.2012.03.009.

[49] G. Karniadakis, A. Beskok, N. Aluru, Microflows and Nanoflows, 2005.

[50] D. Li, Electrokinetics In Microfluidics, Elsevier/Academic Press, 2004.

[51] R. Hunter, Zeta Potential In Colloid Science, Academic Press, 1981.

[52] S. Qian, Y. Ai, Electrokinetic Particle Transport In Micro-/Nanofluidics, CRC Press, 2012.

[53] Y.-J. Oh, A.L. Garcia, D.N. Petsev, G.P. Lopez, S.R. Brueck, C.F. Ivory, S.M. Han, Effect of wall-molecule interactions on electrokinetic transport of charged molecules in nanofluidic channels during FET flow control, Lab Chip 9 (2009) 1601-1608.

[54] D. Jing, B. Bhushan, Electroviscous effect on fluid drag in a microchannel with large zeta potential, Beilstein J. Nanotechnol. 6 (2015) 2207-2216.

[55] B.B. Owen, R.C. Miller, C.E. Milner, H.L. Cogan, The dielectric constant of water as a function of temperature and pressure, J. Phys. Chem. 65 (2007) 2065-2070.

[56] U. Kaatze, Complex permittivity of water as a function of frequency and temperature, J. Chem. Eng. Data 34 (1989) 371-374.
[57] S. Tseng, J.Y. Lin, J.P. Hsu, Theoretical study of temperature influence on the electrophoresis of a pH-regulated polyelectrolyte, Anal. Chim. Acta 847 (2014) 80-89.

[58] B.E. Poling, J.M. Prausnitz, J.P. O'Connel, The properties of gases and liquids Phys. Today 12 (2009).

[59] R. Biswas, B. Bagchi, Limiting ionic conductance of symmetrical, rigid ions in aqueous solutions: temperature dependence and solvent isotope effects, J. Am. Chem. Soc. 119 (1997) 5946-5953.

[60] The physical chemistry of electrolytic solutions, Chem. Eng. News Arch. 36 (2010) 59, https://doi.org/10.1021/cen-v036n004a.p059.

[61] W.M. Haynes, CRC Handbook of Chemistry and Physics, 95th Edition, 2014-2015, 2014. $\langle 10.1136 /$ oem.53.7.504 .

[62] R.A. Robinson, R.H. Stokes, Electrolyte Solutions: Second Revised Edition, 2002.

[63] J. Sefcik, W.A. Goddard, Thermochemistry of silicic acid deprotonation: comparison of gas-phase and solvated DFT calculations to experiment, Geochim. Cosmochim. Acta 65 (2001) 4435-4443.

[64] P. Atkins, J. De Paula, Elements of Physical Chemistry, Oxford University Press, USA, 2013.

[65] a M. Gaudin, D.W. Fuerstenau, Quartz flotation with anionic collectors, Min. Eng. (1955).

[66] J.-P. Hsu, T.-C. Su, P.H. Peng, S.C. Hsu, M.J. Zheng, L.H. Yeh, Unraveling the anomalous surface-charge-dependent osmotic power using a single funnel-shaped nanochannel, ACS Nano 13 (2019) 13374-13381.

[67] X. Liu, R. Tian, R. Li, W. Ding, H. Li, R. Yuan, Principles for the determination of the surface potential of charged particles in mixed electrolyte solutions, Proc. R. Soc. A: Math. Phys. Eng. Sci. 471 (2180) (2015), 20150064.

[68] X. Liu, G. Yang, H. Li, R. Tian, R. Li, X. Jiang, D. Xie, Observation of significant steric, valence and polarization effects and their interplay: a modified theory for electric double layers, RSC Adv. 4 (3) (2014) 1189-1192.

[69] L. Mei, T.H. Chou, Y.S. Cheng, M.J. Huang, L.H. Yeh, S. Qian, Electrophoresis of PH-regulated nanoparticles: impact of the stern layer, Phys. Chem. Chem. Phys. 18 (2016) 9927-9934, https://doi.org/10.1039/c5cp05728k. 